\title{
Equivalence of viscosity and weak solutions for a $p$-parabolic equation
}

\section{JARKKO SILTAKOSKIID}

Abstract. We study the relationship of viscosity and weak solutions to the equation

$$
\partial_{t} u-\Delta_{p} u=f(D u)
$$

where $p>1$ and $f \in C\left(\mathbb{R}^{N}\right)$ satisfies suitable assumptions. Our main result is that bounded viscosity supersolutions coincide with bounded lower semicontinuous weak supersolutions. Moreover, we prove the lower semicontinuity of weak supersolutions when $p \geq 2$.

\section{Introduction}

A classical solution to a partial differential equation is a smooth function that satisfies the equation pointwise. Since many equations that appear in applications admit no such solutions, a more general class of solutions is needed. One such class is the extensively studied distributional weak solutions defined by integration by parts. Another is the celebrated viscosity solutions based on generalized pointwise derivatives. When both classes of solutions can be meaningfully defined, it is naturally crucial that they coincide. This has been profusely studied starting from [10]. In [12], the equivalence of solutions was proved for the parabolic $p$-Laplacian. The objective of the present work is to prove this equivalence in a different way while also allowing the equation to depend on a first-order term. To the best of our knowledge, the proof is new even in the homogeneous case, at least when $1<p<2$.

More precisely, we study the parabolic equation

$$
\partial_{t} u-\Delta_{p} u=f(D u)
$$

where $1<p<\infty$ and $f \in C\left(\mathbb{R}^{N}\right)$ satisfies a certain growth condition, for details see Sect. 2. We show that bounded viscosity supersolutions to (1.1) coincide with bounded lower semicontinuous weak supersolutions. Moreover, we prove the lower

Mathematics Subject Classification: 35K92, 35J60, 35D40, 35D30, 35 B51

Keywords: Comparison principle, Gradient term, Parabolic p-Laplacian, Viscosity solution, Weak solution. 
semicontinuity of weak supersolutions in the range $p \geq 2$ under slightly stronger assumptions on $f$.

To show that viscosity supersolutions are weak supersolutions, we apply the technique introduced by Julin and Juutinen [11]. In contrast to [12], we do not employ the uniqueness machinery of viscosity solutions. Instead, our strategy is to approximate a viscosity supersolution $u$ by its inf-convolution $u_{\varepsilon}$. It is straightforward to show that $u_{\varepsilon}$ is still a viscosity supersolution in a smaller set. This and the pointwise properties of the inf-convolution imply that $u_{\varepsilon}$ is also a weak supersolution in the smaller set. Furthermore, it follows from Caccioppoli's estimates that $u_{\varepsilon}$ converges to $u$ in a suitable Sobolev space. It then remains to pass to the limit to see that $u$ is a weak supersolution.

To show that weak supersolutions are viscosity supersolutions, we apply the argument from [12] that is based on the comparison principle of weak solutions. However, we could not find a reference for comparison principle for Eq. (1.1). Therefore, we give a detailed proof of such a result.

To prove the lower semicontinuity of weak supersolutions, we adapt the strategy of [17]. First, we prove estimates for the essential supremum of a subsolution using Moser's iteration technique. Then, we use those estimates to deduce that a supersolution is lower semicontinuous at its Lebesgue points.

The equivalence of viscosity and weak solutions for the $p$-Laplace equation and its parabolic version was first proven in [12]. A different proof in the elliptic case was found in [11]. Recently the equivalence of solutions has been studied for various equations. These include the normalized $p$-Poisson equation [1], a non-homogeneous $p$-Laplace equation [22] and the normalized $p(x)$-Laplace equation [25]. Moreover, in [24] the equivalence is shown for the radial solutions of a parabolic equation. We also mention that an unpublished version of [18] applies [11] to sketch the equivalence of solutions to (1.1) in the homogeneous case when $p \geq 2$.

Comparison principles for quasilinear parabolic equations have been studied by several authors. In [13], comparison is proven for $\partial_{t} u-\Delta_{p} u+f(u, x, t)=0$ when $p>2$ and $f$ is a continuous function such that $|f(u, x, t)| \leq g(u)$ for some $g \in C^{1}$. The homogeneous case for the $p$-parabolic equation is considered also in [16] and the general equation $\partial_{t} u-\operatorname{div} \mathcal{A}(x, t, D u)=0$ in [15]. Equations with gradient terms are studied for example in [2], where comparison principle is shown for the equation $\partial_{t} u-\Delta_{p} u-|D u|^{\beta}=0$ when $p>2$ and $\beta>p-1$. In the recent papers [4,5], both positive results and counter examples are provided for the comparison, strong comparison, and maximum principles for the equation $\partial_{t} u-\Delta_{p} u-\lambda|u|^{p-2} u-f(x, t)=0$. Furthermore, according to [3], the equation $\partial_{t} u-\Delta_{p} u=q(x)|u|^{\alpha}$ can admit multiple solutions with zero boundary values when $0<\alpha<1$.

The paper is organized as follows. Section 2 contains the precise definitions of weak and viscosity solutions. In Sect. 3, we show that weak supersolutions are viscosity supersolutions, and the converse is shown in Sect. 4. Finally, the lower semicontinuity of weak supersolutions is considered in Sect. 5. 


\section{Preliminaries}

The symbols $\Xi$ and $\Omega$ are reserved for bounded domains in $\mathbb{R}^{N} \times \mathbb{R}$ and $\mathbb{R}^{N}$, respectively. For $t_{1}<t_{2}$, we define the cylinder $\Omega_{t_{1}, t_{2}}:=\Omega \times\left(t_{1}, t_{2}\right)$ and its parabolic boundary $\partial_{p} \Omega_{t_{1}, t_{2}}:=\left(\bar{\Omega} \times\left\{t_{1}\right\}\right) \cup\left(\partial \Omega \times\left(t_{1}, t_{2}\right]\right)$. Moreover, for $T>0$ we set $\Omega_{T}:=\Omega_{0, T}$.

The Sobolev space $W^{1, p}(\Omega)$ contains the functions $u \in L^{p}(\Omega)$ for which the distributional gradient $D u$ exists and belongs in $L^{p}(\Omega)$. It is equipped with the norm

$$
\|u\|_{W^{1, p}(\Omega)}:=\|u\|_{L^{p}(\Omega)}+\|D u\|_{L^{p}(\Omega)} .
$$

A Lebesgue measurable function $u: \Omega_{t_{1}, t_{2}} \rightarrow \mathbb{R}$ belongs to the parabolic Sobolev space $L^{p}\left(t_{1}, t_{2} ; W^{1, p}(\Omega)\right)$ if $u(\cdot, t) \in W^{1, p}(\Omega)$ for almost every $t \in\left(t_{1}, t_{2}\right)$ and the norm

$$
\left(\int_{\Omega_{t_{1}, t_{2}}}|u|^{p}+|D u|^{p} \mathrm{~d} z\right)^{\frac{1}{p}}
$$

is finite. By $d z$, we mean integration with respect to space and time variables, i.e., $d z=d x \mathrm{~d} t$. Integral average is denoted by

$$
f_{\Omega_{T}} u \mathrm{~d} z:=\frac{1}{\left|\Omega_{T}\right|} \int_{\Omega_{T}} u \mathrm{~d} z
$$

Growth condition

Unless otherwise stated, the function $f \in C\left(\mathbb{R}^{N}\right)$ is assumed to satisfy the growth condition

$$
|f(\xi)| \leq C_{f}\left(1+|\xi|^{\beta}\right) \text { for all } \xi \in \mathbb{R}^{N}
$$

where $C_{f}>0$ and $1 \leq \beta<p$.

Definition 2.1. (Weak solution) A function $u: \Xi \rightarrow \mathbb{R}$ is a weak supersolution to (1.1) in $\Xi$ if $u \in L^{p}\left(t_{1}, t_{2} ; W^{1, p}(\Omega)\right)$ whenever $\Omega_{t_{1}, t_{2}} \Subset \Xi$, and

$$
\int_{\Xi}-u \partial_{t} \varphi+|D u|^{p-2} D u \cdot D \varphi-\varphi f(D u) \mathrm{d} z \geq 0
$$

for all non-negative test functions $\varphi \in C_{0}^{\infty}\left(\Omega_{t_{1}, t_{2}}\right)$. For weak subsolutions, the inequality is reversed and a function is a weak solution if it is both a super- and subsolution.

To define viscosity solutions to (1.1), we set for all $\varphi \in C^{2}$ with $D \varphi \neq 0$

$$
\Delta_{p} \varphi:=|D \varphi|^{p-2}\left(\Delta \varphi+\frac{p-2}{|D \varphi|^{2}}\left\langle D^{2} \varphi D \varphi, D \varphi\right\rangle\right) .
$$


Definition 2.2. (Viscosity solution) A lower semicontinuous and bounded function $u: \Xi \rightarrow \mathbb{R}$ is a viscosity supersolution to (1.1) in $\Xi$ if whenever $\varphi \in C^{2}(\Xi)$ and $\left(x_{0}, t_{0}\right) \in \Xi$ are such that

$$
\begin{cases}\varphi\left(x_{0}, t_{0}\right)=u\left(x_{0}, t_{0}\right), & \\ \varphi(x, t)<u(x, t) & \text { when }(x, t) \neq\left(x_{0}, t_{0}\right), \\ D \varphi(x, t) \neq 0 & \text { when } x \neq x_{0},\end{cases}
$$

then

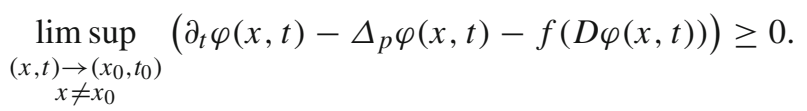

An upper semicontinuous and bounded function $u: \Xi \rightarrow \mathbb{R}$ is a viscosity subsolution to (1.1) in $\Xi$ if whenever $\varphi \in C^{2}(\Xi)$ and $\left(x_{0}, t_{0}\right) \in \Xi$ are such that

$$
\begin{cases}\varphi\left(x_{0}, t_{0}\right)=u\left(x_{0}, t_{0}\right), & \\ \varphi(x, t)>u(x, t) & \text { when }(x, t) \neq\left(x_{0}, t_{0}\right), \\ D \varphi(x, t) \neq 0 & \text { when } x \neq x_{0},\end{cases}
$$

then

$$
\liminf _{\substack{(x, t) \rightarrow\left(x_{0}, t_{0}\right) \\ x \neq x_{0}}}\left(\partial_{t} \varphi(x, t)-\Delta_{p} \varphi(x, t)-f(D \varphi(x, t))\right) \leq 0 .
$$

A function that is both a viscosity sub- and supersolution is a viscosity solution.

If a function $\varphi$ is like in the definition of viscosity supersolution, we say that $\varphi$ touches $u$ from below at $\left(x_{0}, t_{0}\right)$. The limit superior in the definition is needed because the operator $\Delta_{p}$ is singular when $1<p<2$. When $p \geq 2$, the operator is degenerate and the limit superior disappears.

\section{Weak solutions are viscosity solutions}

We show that bounded, lower semicontinuous weak supersolutions to (1.1) are viscosity supersolutions when $1<p<\infty$ and $f \in C\left(\mathbb{R}^{N}\right)$ satisfies the growth condition (G1). One way to prove this kind of results is by applying the comparison principle [12]. However, we could not find the comparison principle for Eq. (1.1) in the literature and therefore we prove it first. To this end, we first prove comparison Lemmas 3.2 and 3.3 for locally Lipschitz continuous $f$. The local Lipschitz continuity allows us to absorb the first-order terms into the terms that appear due to the $p$-Laplacian, see Step 2 in proof of Lemma 3.2. To deal with general $f$, we take a locally Lipschitz continuous approximant $f_{\delta}$ such that $\left\|f-f_{\delta}\right\|_{L^{\infty}\left(\mathbb{R}^{N}\right)}<\delta / 4 T$. Then, for sub- and supersolutions $u$ and $v$, we consider the functions

$$
u_{\delta}:=u-\frac{\delta}{T-t / 2} \text { and } \quad v_{\delta}:=v+\frac{\delta}{T-t / 2} .
$$


These functions will be sub- and supersolutions to (1.1) where $f$ is replaced by $f_{\delta}$. Since $f_{\delta}$ is locally Lipschitz continuous, it follows from Lemmas 3.2 and 3.3 that $u_{\delta} \leq v_{\delta}$. Letting $\delta \rightarrow 0$ then yields that $u \leq v$.

For similar comparison results, see [2, Proposition 2.1] and [13]. See also Chapters 3.5 and 3.6 in [23] for the elliptic case. A minor difference in our results is that instead of requiring that both the subsolution and the supersolution have uniformly bounded gradients, we only require this for the subsolution.

To prove the comparison principle, we need to use a test function that depends on the supersolution itself. However, supersolutions do not necessarily have a time derivative. One way to deal with this is to use mollifications in the time direction. For a compactly supported $\varphi \in L^{p}\left(\Omega_{T}\right)$, we define its time-mollification by

$$
\varphi^{\epsilon}(x, t)=\int_{\mathbb{R}} \varphi(x, t-s) \rho_{\epsilon}(s) \mathrm{d} s,
$$

where $\rho_{\epsilon}$ is a standard mollifier whose support is contained in $(-\epsilon, \epsilon)$. Then, $\varphi^{\epsilon}$ has time derivative and $\varphi^{\epsilon} \rightarrow \varphi$ in $L^{p}\left(\Omega_{T}\right)$. Furthermore, the time-mollification of a supersolution satisfies a regularized equation in the sense of the following lemma.

Lemma 3.1. Let $v \in L^{\infty}\left(\Omega_{T}\right)$ be a weak supersolution (subsolution) to (1.1) in $\Omega_{T}$. Then, we have

$$
\int_{\Omega_{T}}-v^{\epsilon} \partial_{t} \varphi+\left(|D v|^{p-2} D v\right)^{\epsilon} \cdot D \varphi-\varphi(f(D v))^{\epsilon} \mathrm{d} z \geq(\leq) 0
$$

for all $\varphi \in W^{1, p}\left(\Omega_{T}\right) \cap L^{\infty}\left(\Omega_{T}\right)$ with compact support in $\Omega_{T}$. Moreover, if the stronger growth condition

$$
|f(\xi)| \leq C_{f}\left(1+|\xi|^{p-1}\right)
$$

holds, then the assumption $\varphi \in L^{\infty}\left(\Omega_{T}\right)$ is not needed.

Observe that in the above lemma $\varphi$ is in the usual Sobolev space $W^{1, p}\left(\Omega_{T}\right)$ so it has a weak time derivative $\partial_{t} \varphi \in L^{p}\left(\Omega_{T}\right)$. To prove the lemma, one first assumes that $\varphi$ is smooth. Then, testing the weak formulation of (1.1) with $\varphi^{\epsilon}$, changing variables and using Fubini's theorem yields (3.1). The general case follows by approximating $\varphi$ in $W^{1, p}\left(\Omega_{T}\right)$ with the standard mollification. We omit the details.

Lemma 3.2. Let $1<p<2$ and let $f$ be locally Lipschitz. Let $u, v \in L^{\infty}\left(\Omega_{T}\right)$, respectively, be weak sub-and supersolutions to (1.1) in $\Omega_{T}$. Assume that for all $\left(x_{0}, t_{0}\right) \in \partial_{p} \Omega_{T}$

$$
\underset{(x, t) \rightarrow\left(x_{0}, t_{0}\right)}{\operatorname{ess} \lim \sup } u(x, t) \leq \underset{(x, t) \rightarrow\left(x_{0}, t_{0}\right)}{\operatorname{ess} \liminf _{\min }} v(x, t) .
$$

Suppose also that $D u \in L^{\infty}\left(\Omega_{T}\right)$. Then, $u \leq v$ a.e. in $\Omega_{T}$. 
Proof. (Step 1) Let $l>0$ and set $w:=(u-v-l)_{+}$. Let also $s \in(0, T)$. We want to use $w \cdot \chi_{[0, s]}$ as a test function, but since it is not smooth, we must perform mollifications. Let $h>0$ and define

$$
\varphi:=\eta\left((u-v-l)^{\epsilon}\right)_{+},
$$

where

$$
\eta(t)=\left\{\begin{array}{lr}
1, & t \in(0, s-h], \\
(-t+s+h) / 2 h, & t \in(s-h, s+h), \\
0, & t \in[s+h, T) .
\end{array}\right.
$$

The function $\varphi$ is compactly supported and belongs in $W^{1, p}\left(\Omega_{T}\right)$. Therefore, by Lemma 3.1 we have

$$
\begin{aligned}
& \int_{\Omega_{T}}-(u-v)^{\epsilon} \partial_{t} \varphi \mathrm{d} z \\
& \quad \leq \int_{\Omega_{T}}\left(\left(|D v|^{p-2} D v\right)^{\epsilon}-\left(|D u|^{p-2} D u\right)^{\epsilon}\right) \cdot D \varphi+\varphi\left(f(D u)^{\epsilon}-f(D v)^{\epsilon}\right) \mathrm{d} z .
\end{aligned}
$$

We use the linearity of convolution and integration by parts to eliminate the time derivative. We obtain

$$
\begin{aligned}
\int_{\Omega_{T}} & -(u-v)^{\epsilon} \partial_{t} \varphi \mathrm{d} z \\
= & -\int_{\Omega_{T}}(u-v)^{\epsilon}\left((u-v-l)^{\epsilon}\right)_{+} \partial_{t} \eta+\eta(u-v)^{\epsilon} \partial_{t}\left((u-v-l)^{\epsilon}\right)_{+} \mathrm{d} z \\
= & -\int_{\Omega_{T}}(u-v-l)^{\epsilon}\left((u-v-l)^{\epsilon}\right)_{+} \partial_{t} \eta+l\left((u-v-l)^{\epsilon}\right)_{+} \partial_{t} \eta \\
& +\eta(u-v-l)^{\epsilon} \partial_{t}\left((u-v-l)^{\epsilon}\right)_{+}+l \eta \partial_{t}\left((u-v-l)^{\epsilon}\right)_{+} \mathrm{d} z \\
= & -\int_{\Omega_{T}}\left((u-v-l)^{\epsilon}\right)_{+}^{2} \partial_{t} \eta+\frac{1}{2} \eta \partial_{t}\left((u-v-l)^{\epsilon}\right)_{+}^{2} \mathrm{~d} z \\
= & -\frac{1}{2} \int_{\Omega_{T}}\left((u-v-l)^{\epsilon}\right)_{+}^{2} \partial_{t} \eta \mathrm{d} z \\
& \rightarrow-\frac{1}{2} \int_{\Omega_{T}}(u-v-l)_{+}^{2} \partial_{t} \eta \mathrm{d} z
\end{aligned}
$$

Moreover, by the Lebesgue differentiation theorem for a.e. $s \in(0, T)$ it holds

$-\frac{1}{2} \int_{\Omega_{T}}(u-v-l)_{+}^{2} \partial_{t} \eta \mathrm{d} z=\frac{1}{4 h} \int_{s-h}^{s+h} \int_{\Omega} w^{2}(x, t) \mathrm{d} x \mathrm{~d} t \underset{h \rightarrow 0}{\rightarrow} \frac{1}{2} \int_{\Omega} w^{2}(x, s) \mathrm{d} x$.

The terms at the right-hand side of (3.2) converge similarly. Hence, for a.e. $s \in(0, T)$ we have 


$$
\begin{aligned}
& \frac{1}{2} \int_{\Omega} w^{2}(x, s) \mathrm{d} x \\
& \quad \leq \int_{\Omega_{s}}|f(D u)-f(D v)| w \mathrm{~d} z-\int_{\Omega_{s}}\left(|D u|^{p-2} D u-|D v|^{p-2} D v\right) \cdot D w \mathrm{~d} z \\
& \quad=: I_{1}-I_{2}
\end{aligned}
$$

(Step 2) We seek to absorb some of $I_{1}$ into $I_{2}$ so that we can conclude from Grönwall's inequality that $w \equiv 0$ almost everywhere. Since $f$ is locally Lipschitz continuous, there are constants $M \geq \max \left(2\|D u\|_{L^{\infty}\left(\Omega_{T}\right)}, 1\right)$ and $L=L(M)$ such that

$$
|f(\xi)-f(\eta)| \leq L|\xi-\eta| \text { when }|\xi|,|\eta|<M
$$

We denote $\Omega_{s}^{+}:=\left\{x \in \Omega_{s}: w \geq 0\right\}$,

$$
A:=\Omega_{s}^{+} \cap\{|D v|<M\} \text { and } B:=\Omega_{s}^{+} \cap\{|D v| \geq M\} .
$$

Observe that in $B$ we have by the growth condition (G1), choice of $M$ and the assumption that $\beta \geq 1$

$$
|f(D u)| \leq C_{f}\left(1+|D u|^{\beta}\right) \leq C_{f}\left(M+M^{\beta}\right) \leq 2 C_{f} M^{\beta} \leq 2 C_{f}|D v|^{\beta}
$$

and

$$
|f(D v)| \leq C_{f}\left(1+|D v|^{\beta}\right) \leq 2 C_{f}|D v|^{\beta} .
$$

It follows from (3.4), (3.5), (3.6) and Young's inequality that

$$
\begin{aligned}
I_{1} & \leq \int_{A} L|D u-D v| w \mathrm{~d} z+\int_{B}(|f(D u)|+|f(D v)|) w \mathrm{~d} z \\
& \leq \int_{A} L|D u-D v| w \mathrm{~d} z+\int_{B} 4 C_{f}|D v|^{\beta} w \mathrm{~d} z \\
& \leq \int_{A} \epsilon|D u-D v|^{2}+C(\epsilon, L) w^{2} \mathrm{~d} z+\int_{B} \epsilon|D v|^{\frac{\beta p}{\beta}}+C\left(\epsilon, p, \beta, L, C_{f}\right) w^{\frac{p}{p-\beta}} \mathrm{d} z \\
& \leq \epsilon \int_{A}|D u-D v|^{2} \mathrm{~d} z+\epsilon \int_{B}|D v|^{p} \mathrm{~d} z+C\left(\epsilon, p, \beta, L, C_{f},\|w\|_{L^{\infty}}\right) \int_{\Omega_{s}} w^{2} \mathrm{~d} z,
\end{aligned}
$$

where in the last step we used that $\frac{p}{p-\beta}>2$ to estimate

$$
\int_{\Omega_{s}} w^{p /(p-\beta)} \mathrm{d} z=\int_{\Omega_{s}} w^{p /(p-\beta)-2} w^{2} \mathrm{~d} z \leq\|w\|_{L^{\infty}\left(\Omega_{T}\right)}^{p /(p-\beta)-2} \int_{\Omega_{s}} w^{2} \mathrm{~d} z .
$$

Using the vector inequality

$$
\left(|a|^{p-2} a-|b|^{p-2} b\right) \cdot(a-b) \geq(p-1)|a-b|^{2}\left(1+|a|^{2}+|b|^{2}\right)^{\frac{p-2}{2}},
$$


which holds when $1<p<2$ [19, p98], we get

$$
\begin{aligned}
I_{2} & =\int_{\Omega_{s}}\left(|D u|^{p-2} D u-|D v|^{p-2} D v\right) \cdot D w \mathrm{~d} z \\
& \geq(p-1) \int_{\Omega_{s}^{+}} \frac{|D u-D v|^{2}}{\left(1+|D u|^{2}+|D v|^{2}\right)^{\frac{2-p}{2}}} \mathrm{~d} z \\
& \geq(p-1) \int_{A} \frac{|D u-D v|^{2}}{\left(1+M^{2}+M^{2}\right)^{\frac{2-p}{2}}} \mathrm{~d} z+(p-1) \int_{B} \frac{(|D v|-|D u|)^{2}}{\left(3|D v|^{2}\right)^{\frac{2-p}{2}}} \mathrm{~d} z \\
& \geq C(p, M) \int_{A}|D u-D v|^{2} \mathrm{~d} z+(p-1) \int_{B} \frac{\left(|D v|-\frac{1}{2} M\right)^{2}}{\left(3|D v|^{2}\right)^{\frac{2-p}{2}}} \mathrm{~d} z \\
& \geq C(p, M) \int_{A}|D u-D v|^{2} \mathrm{~d} z+(p-1) \int_{B} \frac{\left(\frac{1}{2} \mid D v\right)^{2}}{\left(3|D v|^{2}\right)^{\frac{2-p}{2}}} \mathrm{~d} z \\
& =C(p, M) \int_{A}|D u-D v|^{2} \mathrm{~d} z+C(p) \int_{B}|D v|^{p} \mathrm{~d} z
\end{aligned}
$$

where $C(p, M), C(p)>0$. Combining the estimates (3.7) and (3.9), we arrive at

$$
\begin{aligned}
I_{1}-I_{2} \leq & (\epsilon-C(p, M)) \int_{A}|D u-D v|^{2} \mathrm{~d} z+(\epsilon-C(p)) \int_{B}|D v|^{p} \mathrm{~d} z \\
& +C_{0} \int_{\Omega_{s}} w^{2} \mathrm{~d} z
\end{aligned}
$$

where $C_{0}=C\left(\epsilon, p, \beta, L, C_{f},\|w\|_{L^{\infty}}\right)$. Recalling (3.3) and taking small enough $\epsilon$ yields

$$
\int_{\Omega} w^{2}(x, s) \mathrm{d} x \leq 2 C_{0} \int_{\Omega_{s}} w^{2} \mathrm{~d} z .
$$

Since this holds for a.e. $s \in(0, T)$, Grönwall's inequality implies that $w \equiv 0$ a.e. in $\Omega_{T}$. Finally, letting $l \rightarrow 0$ yields that $u-v \leq 0$ a.e. in $\Omega_{T}$.

Lemma 3.3. Let $p \geq 2$ and let $f$ be locally Lipschitz. Let $v \in L^{\infty}\left(\Omega_{T}\right)$ be a weak supersolution to (1.1) and let $u \in L^{\infty}\left(\Omega_{T}\right)$ be a weak subsolution to

$$
\partial_{t} u-\Delta_{p} u-f(D u) \leq-\delta \text { in } \Omega_{T}
$$

for some $\delta>0$. Assume that for all $\left(x_{0}, t_{0}\right) \in \partial_{p} \Omega_{T}$

$$
\underset{(x, t) \rightarrow\left(x_{0}, t_{0}\right)}{\operatorname{ess} \lim \sup } u(x, t) \leq \underset{(x, t) \rightarrow\left(x_{0}, t_{0}\right)}{\operatorname{ess} \liminf _{\operatorname{ing}}} v(x, t) .
$$

Suppose also that $D u \in L^{\infty}\left(\Omega_{T}\right)$. Then, $u \leq v$ a.e. in $\Omega_{T}$. 
Proof. Let $l>0$ and set $w:=(u-v-l)_{+}$. Let also $s \in(0, T)$. Repeating the first step of the proof of Lemma 3.2, we arrive at the inequality

$$
\begin{aligned}
\frac{1}{2} & \int_{\Omega} w^{2}(x, s) \mathrm{d} x \\
& \leq \int_{\Omega_{s}}|f(D u)-f(D v)| w \mathrm{~d} z-\int_{\Omega_{s}}\left(|D u|^{p-2} D u-|D v|^{p-2} D v\right) \cdot D w \mathrm{~d} z \\
& -\int_{\Omega_{s}} \delta w \mathrm{~d} z \\
= & : I_{1}-I_{2}-\int_{\Omega_{s}} \delta w \mathrm{~d} z .
\end{aligned}
$$

Moreover, we define the constants $M$ and $L$, and the sets $A$ and $B$, exactly in the same way as in the proof of Lemma 3.2. Then, by (3.4), (3.5), (3.6) and Young's inequality

$$
\begin{aligned}
I_{1} & \leq \int_{A} L|D u-D v| w \mathrm{~d} z+\int_{B}(|f(D u)|+|f(D v)|) w \mathrm{~d} z \\
& \leq \int_{A} \epsilon|D u-D v|^{p}+C(\epsilon, L) w^{\frac{p}{p-1}} \mathrm{~d} z+\int_{B} 4 C_{f}|D v|^{\beta} w \mathrm{~d} z \\
& \leq \epsilon \int_{A}|D u-D v|^{p} \mathrm{~d} z+\epsilon \int_{B}|D v|^{p} \mathrm{~d} z+C\left(\epsilon, p, \beta, L, C_{f}\right) \int_{\Omega_{s}} w^{\frac{p}{p-1}} \\
& +w^{\frac{p}{p-\beta}} \mathrm{d} z .
\end{aligned}
$$

Using the vector inequality

$$
\left(|a|^{p-2} a-|b|^{p-2} b\right) \cdot(a-b) \geq 2^{2-p}|a-b|^{p},
$$

which holds when $p \geq 2$ [19, p. 95], we get

$$
I_{2} \geq C(p) \int_{A}|D u-D v|^{p} \mathrm{~d} z+C(p) \int_{B}|D u-D v|^{p} \mathrm{~d} z .
$$

Furthermore, since in $B$ it holds

$$
|D u-D v|^{p} \geq(|D v|-|D u|)^{p} \geq\left(|D v|-\frac{1}{2} M\right)^{p} \geq C(p)|D v|^{p},
$$

we arrive at

$$
I_{2} \geq C(p) \int_{A}|D u-D v|^{p} \mathrm{~d} z+C(p) \int_{B}|D v|^{p} \mathrm{~d} z .
$$

Combining (3.11) and (3.13) with (3.10), we get

$$
\begin{aligned}
\frac{1}{2} \int_{\Omega} w^{2} \mathrm{~d} x \leq & (\epsilon-C(p))\left(\int_{A}|D u-D v|^{p} \mathrm{~d} z+\int_{B}|D v|^{p} \mathrm{~d} z\right) \\
& +\int_{\Omega_{s}} C\left(\epsilon, p, \beta, L, C_{f}\right)\left(w^{\frac{p}{p-1}}+w^{\frac{p}{p-\beta}}\right)-\delta w \mathrm{~d} z .
\end{aligned}
$$


By taking small enough $\epsilon=\epsilon(p)$, the above becomes

$$
\int_{\Omega} w^{2}(x, s) \mathrm{d} x \leq \int_{\Omega_{s}} C\left(p, \beta, L, C_{f}\right)\left(w^{\frac{p}{p-1}}+w^{\frac{p}{p-\beta}}\right)-\delta w \mathrm{~d} z .
$$

Observe that since $w$ is bounded and $\frac{p}{p-1}, \frac{p}{p-\beta}>1$, the integrand at the right-hand side is bounded by some constant times $w^{2}$. To argue this rigorously, we write down the following algebraic fact.

If $a_{0}, \delta, \gamma>0$ and $\alpha>1$, then there exists $C\left(\alpha, \gamma, \delta, a_{0}\right)>0$ such that

$$
\gamma a^{\alpha} \leq \delta a+C\left(\alpha, \gamma, \delta, a_{0}\right) a^{2} \text { for all } a \in\left[0, a_{0}\right) .
$$

To see this, let first $\alpha<2$. Then, by Young's inequality

$$
\begin{aligned}
\gamma a^{\alpha}=\gamma a \cdot a^{\alpha-1} & \leq \frac{\delta}{1+a_{0}^{\frac{2}{3-\alpha}}} a^{\frac{2}{3-\alpha}}+C\left(\alpha, \gamma, \delta, a_{0}\right) a^{(\alpha-1) \cdot \frac{2}{\alpha-1}} \\
& \leq \delta a+C\left(\alpha, \gamma, \delta, a_{0}\right) a^{2}
\end{aligned}
$$

If $\alpha \geq 2$, then

$$
\gamma a^{\alpha}=\gamma a^{\alpha-2} \cdot a^{2} \leq \gamma a_{0}^{\alpha-2} a^{2} .
$$

Applying the algebraic fact on (3.14), we get

$$
\int_{\Omega} w^{2}(x, s) \mathrm{d} x \leq C\left(p, \beta, L, C_{f}, \delta,\|w\|_{L^{\infty}}\right) \int_{\Omega_{s}} w^{2} \mathrm{~d} z .
$$

The conclusion now follows from Grönwall's inequality and letting $l \rightarrow 0$.

Next, we use the previous comparison results to prove the comparison principle for general continuous $f$.

Theorem 3.4. Let $1<p<\infty$. Let $u, v \in L^{\infty}\left(\Omega_{T}\right)$, respectively, be weak sub-and supersolutions to (1.1) in $\Omega_{T}$. Assume that for all $\left(x_{0}, t_{0}\right) \in \partial_{p} \Omega_{T}$

$$
\underset{(x, t) \rightarrow\left(x_{0}, t_{0}\right)}{\operatorname{ess} \lim \sup } u(x, t) \leq \underset{(x, t) \rightarrow\left(x_{0}, t_{0}\right)}{\operatorname{ess} \liminf _{\operatorname{mon}}} v(x, t) .
$$

Assume also that $D u \in L^{\infty}\left(\Omega_{T}\right)$. Then, $u \leq v$ a.e. in $\Omega_{T}$.

Proof. For $\delta>0$, define

$$
u_{\delta}:=u-\frac{\delta}{T-t / 2} .
$$

Then, for any non-negative test function $\varphi \in C_{0}^{\infty}\left(\Omega_{T}\right)$ we have by integration by parts

$$
\int_{\Omega_{T}}-u_{\delta} \partial_{t} \varphi \mathrm{d} z=\int_{\Omega_{T}}-u \partial_{t} \varphi+\frac{\delta}{T-t / 2} \partial_{t} \varphi \mathrm{d} z
$$




$$
\begin{aligned}
& =\int_{\Omega_{T}}-u \partial_{t} \varphi-\varphi \frac{\delta}{2(T-t / 2)^{2}} \mathrm{~d} z \\
& \leq \int_{\Omega_{T}}-u \partial_{t} \varphi-\varphi \frac{\delta}{2 T^{2}} \mathrm{~d} z .
\end{aligned}
$$

Since $f$ is continuous, there is a locally Lipschitz continuous function $f_{\delta}$ such that $\left\|f-f_{\delta}\right\|_{L^{\infty}\left(\mathbb{R}^{N}\right)} \leq \frac{\delta}{4 T}$ (see, e.g., [21]). Then, since $u$ is a weak subsolution, we have

$$
\begin{aligned}
& \int_{\Omega_{T}}-u_{\delta} \partial_{t} \varphi+\left|D u_{\delta}\right|^{p-2} D u_{\delta} \cdot D \varphi-\varphi f_{\delta}\left(D u_{\delta}\right) \mathrm{d} z \\
& \quad \leq \int_{\Omega_{T}}-u \partial_{t} \varphi+|D u|^{p-2} D u \cdot D \varphi-\varphi f(D u)+\varphi\left\|f-f_{\delta}\right\|_{L^{\infty}\left(\mathbb{R}^{\mathbb{N}}\right)}-\varphi \frac{\delta}{2 T^{2}} \mathrm{~d} z \\
& \quad \leq \int_{\Omega_{T}}-\frac{\delta}{4 T^{2}} \varphi \mathrm{d} z .
\end{aligned}
$$

Hence, $u_{\delta}$ is a weak subsolution to

$$
\partial_{t} u_{\delta}-\Delta_{p} u_{\delta}-f_{\delta}\left(D u_{\delta}\right) \leq-\frac{\delta}{4 T^{2}} \text { in } \Omega_{T} .
$$

Similarly, since $v$ is a weak supersolution, we define

$$
v_{\delta}:=v+\frac{\delta}{T-t / 2}
$$

and deduce that $v_{\delta}$ is a weak supersolution to

$$
\partial_{t} v_{\delta}-\Delta_{p} v_{\delta}-f_{\delta}\left(D v_{\delta}\right) \geq 0 \text { in } \Omega_{T} .
$$

Now it follows from the comparison Lemmas 3.2 and 3.3 that $u_{\delta} \leq v_{\delta}$ a.e. in $\Omega_{T}$. Thus,

$$
u \leq v+\frac{2 \delta}{T-t / 2} \quad \text { a.e. in } \Omega_{T} .
$$

Letting $\delta \rightarrow 0$ finishes the proof.

Now that the comparison principle is proven, we are ready to show that weak solutions are viscosity solutions. To state this part of the equivalence, we define the lower semicontinuous regularization of a function $u: \Xi \rightarrow \mathbb{R}$ by

$$
u_{*}(x, t):=\underset{(y, s) \rightarrow(x, t)}{\operatorname{ess} \liminf } u(y, s):=\lim _{R \rightarrow 0} \underset{B_{R}(x) \times\left(t-R^{p}, t+R^{p}\right)}{\operatorname{essinf}} u .
$$

The time scaling $R^{p}$ is technically convenient in Sect. 5. We have included it here for notational consistency.

Theorem 3.5. Let $1<p<\infty$. Let $u \in L_{\text {loc }}^{\infty}(\Xi)$ be a weak supersolution to (1.1) in $\Xi$ for which $u=u_{*}$ almost everywhere in $\Xi$. Then, $u_{*}$ is a viscosity supersolution to (1.1) in $\Xi$. 
Proof. Assume on the contrary that there is $\phi \in C^{2}(\Xi)$ touching $u_{*}$ from below at $\left(x_{0}, t_{0}\right) \in \Xi, D \phi(x, t) \neq 0$ for $x \neq x_{0}$ and

$$
\limsup _{\substack{(x, t) \rightarrow\left(x_{0}, t_{0}\right) \\ x \neq x_{0}}}\left(\partial_{t} \phi(x, t)-\Delta_{p} \phi(x, t)-f(D \phi(x, t))\right)<0 .
$$

Denote $Q_{r}:=B_{r}\left(x_{0}\right) \times\left(t_{0}-r, t_{0}+r\right)$. It follows from above that there are $r>0$ and $\delta>0$ such that

$$
\partial_{t} \phi-\Delta_{p} \phi-f(D \phi)<-\delta \text { in } Q_{r} \backslash\left\{x=x_{0}\right\} .
$$

Indeed, otherwise there would be a sequence $\left(x_{n}, t_{n}\right) \rightarrow\left(x_{0}, t_{0}\right)$ such that $x_{n} \neq x_{0}$ and

$$
\partial_{t} \phi\left(x_{n}, t_{n}\right)-\Delta_{p} \phi\left(x_{n}, t_{n}\right)-f\left(D \phi\left(x_{n}, t_{n}\right)\right)>-\frac{1}{n},
$$

but this contradicts (3.15). Using Gauss's theorem and (3.16), we obtain for any nonnegative test function $\varphi \in C_{0}^{\infty}\left(Q_{r}\right)$ that

$$
\begin{aligned}
& \int_{Q_{r}}-\phi \partial_{t} \varphi+|D \phi|^{p-2} D \phi \cdot D \varphi-\varphi f(D \phi) \mathrm{d} z \\
& \quad=\lim _{\rho \rightarrow 0} \int_{Q_{r} \backslash\left\{\left|x-x_{0}\right| \leq \rho\right\}}-\phi \partial_{t} \varphi+|D \phi|^{p-2} D \phi \cdot D \varphi-\varphi f(D \phi) \mathrm{d} z \\
& =\lim _{\rho \rightarrow 0}\left(\int_{Q_{r} \backslash\left\{\left|x-x_{0}\right| \leq \rho\right\}} \varphi \partial_{t} \phi-\varphi \operatorname{div}\left(|D \phi|^{p-2} D \phi\right)-\varphi f(D \phi) \mathrm{d} z\right. \\
& \left.\quad+\int_{t_{0}-r}^{t_{0}+r} \int_{\left\{\left|x-x_{0}\right|=\rho\right\}} \varphi|D \phi|^{p-2} D \phi \cdot \frac{\left(x-x_{0}\right)}{\rho} \mathrm{d} S \mathrm{~d} t\right) \\
& =\lim _{\rho \rightarrow 0} \int_{Q_{r} \backslash\left\{\left|x-x_{0}\right| \leq \rho\right\}} \varphi\left(\partial_{t} \phi-\Delta_{p} \phi-f(D \phi)\right) \mathrm{d} z \\
& \leq \int_{Q_{r}}-\delta \varphi \mathrm{d} z .
\end{aligned}
$$

Let $l:=\min _{\partial_{p} Q_{r}}\left(u_{*}-\phi\right)>0$ and set $\widetilde{\phi}:=\phi+l$. Then, by the above inequality, $\widetilde{\phi}$ is a weak subsolution to

$$
\partial_{t} \widetilde{\phi}-\Delta_{p} \widetilde{\phi}-f(D \widetilde{\phi}) \leq-\delta \text { in } Q_{r}
$$

and on $\partial_{p} Q_{r}$ it holds $\widetilde{\phi}=\phi+l \leq \phi+u_{*}-\phi=u_{*}$. Hence, Theorem 3.4 implies that $\widetilde{\phi} \leq u$ almost everywhere in $Q_{r}$. By the definition of $u_{*}$, it follows that

$$
\widetilde{\phi} \leq u_{*} \text { everywhere in } Q_{r}
$$

which is a contradiction since in particular $\widetilde{\phi}\left(x_{0}, t_{0}\right)=\phi\left(x_{0}, t_{0}\right)+l>u_{*}\left(x_{0}, t_{0}\right)$.

To see (3.17), fix $\left(y_{0}, s_{0}\right) \in Q_{r}$ and let $\varepsilon>0$. By continuity of $\widetilde{\phi}$ and the definition of $u_{*}$, there is $R>0$ such that

$$
\left|\widetilde{\phi}(y, s)-\widetilde{\phi}\left(y_{0}, s_{0}\right)\right| \leq \varepsilon \text { for all }(y, s) \in Q_{R}^{\prime}
$$


and

$$
\left|\underset{Q_{R}^{\prime}}{\operatorname{ess} \inf } u-u_{*}\left(y_{0}, s_{0}\right)\right|<\varepsilon
$$

where we denoted $Q_{R}^{\prime}:=B_{R}\left(y_{0}\right) \times\left(s_{0}-R^{p}, s_{0}+R^{p}\right)$. In particular,

$$
u_{*}\left(y_{0}, s_{0}\right) \geq \underset{Q_{R}^{\prime}}{\operatorname{essinf}} u-\varepsilon .
$$

By the definition of essinf $u$, there is $A \subset Q_{R}^{\prime}$ with $|A|>0$ such that

$$
\underset{Q_{R}^{\prime}}{\operatorname{essinf}} u+\varepsilon>u(y, s) \text { for all }(y, s) \in A .
$$

Moreover, since $\widetilde{\phi} \leq u$ almost everywhere in $Q_{r}$, we can take $(y, s) \in A$ such that

$$
\widetilde{\phi}(y, s) \leq u(y, s) .
$$

Now we have by the last three displays

$$
u_{*}\left(y_{0}, s_{0}\right) \geq \underset{Q_{R}^{\prime}}{\operatorname{essinf}} u-\varepsilon>u(y, s)-2 \varepsilon \geq \widetilde{\phi}(y, s)-2 \varepsilon \geq \widetilde{\phi}\left(y_{0}, s_{0}\right)-3 \varepsilon .
$$

Since $\varepsilon>0$ was arbitrary, this implies that $u_{*}\left(y_{0}, s_{0}\right) \geq \widetilde{\phi}\left(y_{0}, s_{0}\right)$.

\section{Viscosity solutions are weak solutions}

We show that bounded viscosity supersolutions to (1.1) are weak supersolutions when $1<p<\infty$ and $f \in C\left(\mathbb{R}^{N}\right)$ satisfies the growth condition (G1). We use the method developed in [11]. The method of [11] was previously applied to parabolic equations in [24], but for radially symmetric solutions.

The idea is to approximate a viscosity supersolution $u$ to (1.1) by the inf-convolution

$$
u_{\varepsilon}(x, t):=\inf _{(y, s) \in \Xi}\left\{u(y, s)+\frac{|x-y|^{q}}{q \varepsilon^{q-1}}+\frac{|t-s|^{2}}{2 \varepsilon}\right\},
$$

where $\varepsilon>0$ and $q \geq 2$ is a fixed constant so large that $p-2+\frac{q-2}{q-1}>0$. If $u$ is bounded, it is straightforward to show that the inf-convolution $u_{\varepsilon}$ is a viscosity supersolution in the smaller set

$$
\Xi_{\varepsilon}=\left\{(x, t) \in \Xi: B_{r(\varepsilon)}(x) \times(t-t(\varepsilon), t+t(\varepsilon)) \Subset \Xi\right\},
$$

where $r(\varepsilon), t(\varepsilon) \rightarrow 0$ as $\varepsilon \rightarrow 0$. Moreover, $u_{\varepsilon}$ is semi-concave by definition and therefore it has a second derivative almost everywhere. It follows from these pointwise properties that $u_{\varepsilon}$ is a weak supersolution to (1.1) in $\Xi_{\varepsilon}$. Caccioppoli-type estimates then imply that $u_{\varepsilon}$ converges to $u$ in a parabolic Sobolev space and consequently $u$ is a weak supersolution. 
The standard properties of the inf-convolution are postponed to the end of this section. Instead, we begin by proving the key observation: that the inf-convolution of a viscosity supersolution is a weak supersolution in the smaller set $\Xi_{\varepsilon}$. When $p \geq 2$, the idea is the following. Since $u_{\varepsilon}$ is a viscosity supersolution to (1.1) that is twice differentiable almost everywhere, it satisfies the equation pointwise almost everywhere. Hence, we may multiply the equation by a non-negative test function $\varphi$ and integrate over $\Xi_{\varepsilon}$ so that the integral will be non-negative. Then, we approximate this expression through smooth functions $u_{\varepsilon, j}$ defined via the standard mollification. Since $u_{\varepsilon, j}$ is smooth, we may integrate by parts to reach the weak formulation of the equation, see (4.1). It then remains to let $j \rightarrow \infty$ to conclude that $u_{\varepsilon}$ is a weak supersolution. The range $1<p<2$ is more delicate because of the singularity of the $p$-Laplace operator

$$
\Delta_{p} u:=|D u|^{p-2}\left(\Delta u+\frac{(p-2)}{|D u|^{2}}\left\langle D^{2} u D u, D u\right\rangle\right),
$$

and therefore we consider the case $p \geq 2$ first.

Lemma 4.1. Let $p \geq 2$. Let $u$ be a bounded viscosity supersolution to (1.1) in $\Xi$. Then, $u_{\varepsilon}$ is a weak supersolution to (1.1) in $\Xi_{\varepsilon}$.

Proof. Fix a non-negative test function $\varphi \in C_{0}^{\infty}\left(\Xi_{\varepsilon}\right)$. By Remark 4.8, the function

$$
\phi(x, t):=u_{\varepsilon}(x, t)-C(q, \varepsilon, u)\left(|x|^{2}+t^{2}\right)
$$

is concave in $\Xi_{\varepsilon}$ and we can approximate it by smooth concave functions $\phi_{j}$ so that $\left(\phi_{j}, \partial_{t} \phi_{j}, D \phi_{j}, D^{2} \phi_{j}\right) \rightarrow\left(\phi, \partial_{t} \phi, D \phi, D^{2} \phi\right)$ a.e. in $\Xi_{\varepsilon}$. We define

$$
u_{\varepsilon, j}(x, t):=\phi_{j}(x, t)+C(q, \varepsilon, u)\left(|x|^{2}+t^{2}\right) .
$$

Since $u_{\varepsilon, j}$ is smooth and $\varphi$ is compactly supported in $\Xi_{\varepsilon}$, we integrate by parts to get

$$
\begin{aligned}
& \int_{\Xi_{\varepsilon}} \varphi\left(\partial_{t} u_{\varepsilon, j}-\left|D u_{\varepsilon, j}\right|^{p-2}\left(\Delta u_{\varepsilon, j}+\frac{(p-2)}{\left|D u_{\varepsilon, j}\right|^{2}}\left\langle D^{2} u_{\varepsilon, j} D u_{\varepsilon, j}, D u_{\varepsilon, j}\right\rangle\right)\right. \\
& \left.\quad-f\left(D u_{\varepsilon, j}\right)\right) \mathrm{d} z \\
& =\int_{\Xi_{\varepsilon}} \varphi \partial_{t} u_{\varepsilon, j}-\varphi \operatorname{div}\left(\left|D u_{\varepsilon, j}\right|^{p-2} D u_{\varepsilon, j}\right)-\varphi f\left(D u_{\varepsilon, j}\right) \mathrm{d} z \\
& =\int_{\Xi_{\varepsilon}}-u_{\varepsilon, j} \partial_{t} \varphi+\left|D u_{\varepsilon, j}\right|^{p-2} D u_{\varepsilon, j} \cdot D \varphi-\varphi f\left(D u_{\varepsilon, j}\right) \mathrm{d} z
\end{aligned}
$$

This implies that

$$
\liminf _{j \rightarrow \infty} \int_{\Xi_{\varepsilon}} \varphi\left(\partial_{t} u_{\varepsilon, j}-\left|D u_{\varepsilon, j}\right|^{p-2}\left(\Delta u_{\varepsilon, j}+\frac{(p-2)}{\left|D u_{\varepsilon, j}\right|^{2}}\left\langle D^{2} u_{\varepsilon, j} D u_{\varepsilon, j}, D u_{\varepsilon, j}\right\rangle\right)\right.
$$




$$
\begin{aligned}
& \left.-f\left(D u_{\varepsilon, j}\right)\right) \mathrm{d} z \\
\leq & \lim _{j \rightarrow \infty} \int_{\Xi_{\varepsilon}}-u_{\varepsilon, j} \partial_{t} \varphi+\left|D u_{\varepsilon, j}\right|^{p-2} D u_{\varepsilon, j} \cdot D \varphi-\varphi f\left(D u_{\varepsilon, j}\right) \mathrm{d} z .
\end{aligned}
$$

We intend to use Fatou's lemma at the left-hand side and dominated convergence at the right-hand side. Once we verify their assumptions, we arrive at the inequality

$$
\begin{aligned}
& \int_{\Xi_{\varepsilon}} \varphi\left(\partial_{t} u_{\varepsilon}-\Delta_{p} u_{\varepsilon}-f\left(D u_{\varepsilon}\right)\right) \mathrm{d} z \leq \int_{\Xi_{\varepsilon}}-u_{\varepsilon} \partial_{t} \varphi \\
& \quad+\left|D u_{\varepsilon}\right|^{p-2} D u_{\varepsilon} \cdot D \varphi-\varphi f\left(D u_{\varepsilon}\right) \mathrm{d} z .
\end{aligned}
$$

The left-hand side is non-negative since by Lemma 4.7 the inf-convolution $u_{\varepsilon}$ is still a viscosity supersolution in $\Xi_{\varepsilon}$. Consequently $u_{\varepsilon}$ is a weak supersolution in $\Xi_{\varepsilon}$ as desired. It remains to justify our use of Fatou's lemma and the dominated convergence theorem. It follows from Remark 4.8 that $\left|u_{\varepsilon, j}\right|,\left|\partial_{t} u_{\varepsilon, j}\right|$ and $\left|D u_{\varepsilon, j}\right|$ are uniformly bounded by some constant $M>0$ in the support of $\varphi$ with respect to $j$. This justifies our use of the dominated convergence theorem. Observe then that since $\phi_{j}$ is concave, we have $D^{2} u_{\varepsilon, j} \leq C(q, \varepsilon, u) I$. Hence,

$$
\begin{gathered}
\partial_{t} u_{\varepsilon, j}-\left|D u_{\varepsilon, j}\right|^{p-2}\left(\Delta u_{\varepsilon, j}+\frac{(p-2)}{\left|D u_{\varepsilon, j}\right|^{2}}\left\langle D^{2} u_{\varepsilon, j} D u_{\varepsilon, j}, D u_{\varepsilon, j}\right\rangle\right)-f\left(D u_{\varepsilon, j}\right) \\
\geq-M-C(q, \varepsilon, u) M^{p-2}(N+p-2)-\sup _{|\xi| \leq M}|f(\xi)| .
\end{gathered}
$$

The integrand at the left-hand side of (4.1) is therefore bounded from below with respect to $j$, justifying our use of Fatou's lemma.

Next, we consider the singular case $1<p<2$. We cannot directly repeat the previous proof because $\Delta_{p} u_{\varepsilon}$ no longer has a clear meaning at the points where $D u_{\varepsilon}=0$. To deal with this, we consider the regularized terms

$$
\Delta_{p, \delta} u:=\left(\delta+|D u|^{2}\right)^{\frac{p-2}{2}}\left(\Delta u+\frac{p-2}{\delta+|D u|^{2}} \Delta_{\infty} u\right),
$$

where $\Delta_{\infty} u=\left\langle D^{2} u D u, D u\right\rangle$.

Lemma 4.2. Let $1<p<2$. Let $u$ be a bounded viscosity supersolution to (1.1) in $\Xi$. Then, $u_{\varepsilon}$ is a weak supersolution to (1.1) in $\Xi_{\varepsilon}$.

Proof. (Step 1) Let $\varphi \in C_{0}^{\infty}\left(\Xi_{\varepsilon}\right)$ be a non-negative test function. We set

$$
\phi(x, t):=u_{\varepsilon}(x, t)-C(q, \varepsilon, u)\left(|x|^{2}+t^{2}\right),
$$

where $C(q, \varepsilon, u)$ is the semi-concavity constant of $u_{\varepsilon}$ in $\Xi_{\varepsilon}$. Then, by Remark 4.8 we can approximate $\phi$ by smooth concave functions $\phi_{j}$ so that $\left(\phi_{j}, \partial_{t} \phi_{j}, D \phi_{j}, D^{2} \phi_{j}\right) \rightarrow$ $\left(\phi, \partial_{t} \phi, D \phi, D^{2} \phi\right)$ a.e. in $\Xi_{\varepsilon}$. We define

$$
u_{\varepsilon, j}(x, t):=\phi_{j}(x, t)+C(q, \varepsilon, u)\left(|x|^{2}+t^{2}\right) .
$$


Let $\delta \in(0,1)$. Since $u_{\varepsilon, j}$ is smooth and $\varphi$ is compactly supported in $\boldsymbol{\Xi}_{\varepsilon}$, we calculate via integration by parts

$$
\begin{aligned}
& \int_{\Xi_{\varepsilon}} \varphi\left(\partial_{t} u_{\varepsilon, j}-\left(\delta+\left|D u_{\varepsilon, j}\right|^{2}\right)^{\frac{p-2}{2}}\left(\Delta u_{\varepsilon, j}+\frac{p-2}{\delta+\left|D u_{\varepsilon, j}\right|^{2}} \Delta_{\infty} u_{\varepsilon, j}\right)-f\left(D u_{\varepsilon, j}\right)\right) \mathrm{d} z \\
& =\int_{\Xi_{\varepsilon}} \varphi \partial_{t} u_{\varepsilon, j}-\varphi \operatorname{div}\left(\left(\delta+\left|D u_{\varepsilon, j}\right|^{2}\right)^{\frac{p-2}{2}} D u_{\varepsilon, j}\right)-\varphi f\left(D u_{\varepsilon, j}\right) \mathrm{d} z \\
& =\int_{\Xi_{\varepsilon}}-u_{\varepsilon, j} \partial_{t} \varphi+\left(\delta+\left|D u_{\varepsilon, j}\right|^{2}\right)^{\frac{p-2}{2}} D u_{\varepsilon, j} \cdot D \varphi-\varphi f\left(D u_{\varepsilon, j}\right) \mathrm{d} z .
\end{aligned}
$$

Recalling the shorthand $\Delta_{p, \delta}$ defined in (4.2), we deduce from the above that

$$
\begin{aligned}
& \liminf _{j \rightarrow \infty} \int_{\Xi_{\varepsilon}} \varphi\left(\partial_{t} u_{\varepsilon, j}-\Delta_{p, \delta} u_{\varepsilon, j}-f\left(D u_{\varepsilon, j}\right)\right) \mathrm{d} z \\
& \quad \leq \lim _{j \rightarrow \infty} \int_{\Xi_{\varepsilon}}-u_{\varepsilon, j} \partial_{t} \varphi+\left(\delta+\left|D u_{\varepsilon, j}\right|^{2}\right)^{\frac{p-2}{2}} D u_{\varepsilon, j} \cdot D \varphi-\varphi f\left(D u_{\varepsilon, j}\right) \mathrm{d} z
\end{aligned}
$$

We use Fatou's lemma at the left-hand side and the dominated convergence at the righthand side. Once we verify their assumptions, we arrive at the auxiliary inequality

$$
\begin{aligned}
& \int_{\Xi_{\varepsilon}} \varphi\left(\partial_{t} u_{\varepsilon}-\Delta_{p, \delta} u_{\varepsilon}-f\left(D u_{\varepsilon}\right)\right) \mathrm{d} z \\
& \quad \leq \int_{\Xi_{\varepsilon}}-u_{\varepsilon} \partial_{t} \varphi+\left(\delta+\left|D u_{\varepsilon}\right|^{2}\right)^{\frac{p-2}{2}} D u_{\varepsilon} \cdot D \varphi-\varphi f\left(D u_{\varepsilon}\right) \mathrm{d} z .
\end{aligned}
$$

Next, we verify the assumptions of Fatou's lemma and the dominated convergence theorem. By Remark 4.8, the functions $\left|u_{\varepsilon, j}\right|,\left|\partial_{t} u_{\varepsilon, j}\right|$ and $\left|D u_{\varepsilon, j}\right|$ are uniformly bounded by some constant $M>1$ in the support of $\varphi$ with respect to $j$. Hence, the assumptions of the dominated convergence theorem are satisfied. Observe then that the concavity of $\phi_{j}$ implies that $D^{2} u_{\varepsilon, j} \leq C(q, \varepsilon, u) I$. Thus, the integrand at the left-hand side of (4.3) has a lower bound independent of $j$ when $D u_{\varepsilon, j}=0$. When $D u_{\varepsilon, j} \neq 0$, we have

$$
\begin{aligned}
\partial_{t} u_{\varepsilon, j}-\left(\delta+\left|D u_{\varepsilon, j}\right|^{2}\right)^{\frac{p-2}{2}}\left(\Delta u_{\varepsilon, j}+\frac{p-2}{\delta+\left|D u_{\varepsilon, j}\right|^{2}} \Delta_{\infty} u_{\varepsilon, j}\right)-f\left(D u_{\varepsilon, j}\right) \\
=\partial_{t} u_{\varepsilon, j}-\frac{\left(\delta+\left|D u_{\varepsilon, j}\right|^{2}\right)^{\frac{p-2}{2}}}{\delta+\left|D u_{\varepsilon, j}\right|^{2}}\left(\left|D u_{\varepsilon, j}\right|^{2}\left(\Delta u_{\varepsilon, j}+\frac{p-2}{\left|D u_{\varepsilon, j}\right|^{2}} \Delta_{\infty} u_{\varepsilon, j}\right)+\delta \Delta u_{\varepsilon, j}\right) \\
\quad-f\left(D u_{\varepsilon, j}\right) \\
\geq-\partial_{t} u_{\varepsilon, j}-\frac{\left(\delta+\left|D u_{\varepsilon, j}\right|^{2}\right)^{\frac{p-2}{2}}}{\delta+\left|D u_{\varepsilon, j}\right|^{2}} C(q, \varepsilon, u)\left(\left|D u_{\varepsilon, j}\right|^{2}(N+p-2)+\delta N\right)-f\left(D u_{\varepsilon, j}\right) \\
\geq-\partial_{t} u_{\varepsilon, j}-C(q, \varepsilon, u)\left(\delta+\left|D u_{\varepsilon, j}\right|^{2}\right)^{\frac{p-2}{2}}(2 N+p-2)-f\left(D u_{\varepsilon, j}\right) \\
\geq-M-C(q, \varepsilon, u) \delta^{\frac{p-2}{2}}(2 N+p-2)-\sup _{|\xi| \leq M}|f(\xi)|,
\end{aligned}
$$


so that our use of Fatou's lemma is justified.

(Step 2) We let $\delta \rightarrow 0$ in the auxiliary inequality (4.4). Since $u_{\varepsilon}$ is Lipschitz continuous, the dominated convergence theorem implies

$$
\begin{aligned}
& \liminf _{\delta \rightarrow 0} \int_{\Xi_{\varepsilon}} \varphi\left(\partial_{t} u_{\varepsilon}-\Delta_{p, \delta} u_{\varepsilon}-f\left(D u_{\varepsilon}\right)\right) \mathrm{d} z \\
& \quad \leq \int_{\Xi_{\varepsilon}}-u_{\varepsilon} \partial_{t} \varphi+\left|D u_{\varepsilon}\right|^{p-2} D u_{\varepsilon} \cdot D \varphi-\varphi f\left(D u_{\varepsilon}\right) \mathrm{d} z .
\end{aligned}
$$

Applying Fatou's lemma (we verify assumptions at the end), we get

$$
\begin{aligned}
\liminf _{\delta \rightarrow 0} \int_{\Xi_{\varepsilon}} \varphi\left(\partial_{t} u_{\varepsilon}-\Delta_{p, \delta} u_{\varepsilon}-f\left(D u_{\varepsilon}\right)\right) \mathrm{d} z \\
\geq \int_{\Xi_{\varepsilon}} \liminf _{\delta \rightarrow 0} \varphi\left(\partial_{t} u_{\varepsilon}-\Delta_{p, \delta} u_{\varepsilon}-f\left(D u_{\varepsilon}\right)\right) \mathrm{d} z \\
=\int_{\Xi_{\varepsilon} \cap\left\{D u_{\varepsilon} \neq 0\right\}} \liminf _{\delta \rightarrow 0} \varphi\left(\partial_{t} u_{\varepsilon}-\Delta_{p, \delta} u_{\varepsilon}-f\left(D u_{\varepsilon}\right)\right) \mathrm{d} z \\
\quad+\int_{\Xi_{\varepsilon} \cap\left\{D u_{\varepsilon}=0\right\}} \liminf _{\delta \rightarrow 0} \varphi\left(\partial_{t} u_{\varepsilon}-\delta^{\frac{p-2}{2}} \Delta u_{\varepsilon}-f(0)\right) \mathrm{d} z \\
=\int_{\Xi_{\varepsilon} \cap\left\{D u_{\varepsilon} \neq 0\right\}} \varphi\left(\partial_{t} u_{\varepsilon}-\Delta_{p} u_{\varepsilon}-f\left(D u_{\varepsilon}\right)\right) \mathrm{d} z \\
\quad+\int_{\Xi_{\varepsilon} \cap\left\{D u_{\varepsilon}=0\right\}} \varphi\left(\partial_{t} u_{\varepsilon}-f(0)\right) \mathrm{d} z \geq 0,
\end{aligned}
$$

where the last inequality follows from Lemma 4.7 since $u_{\varepsilon}$ is twice differentiable almost everywhere. Combining (4.5) and (4.6), we find that $u_{\varepsilon}$ is a weak supersolution in $\Xi_{\varepsilon}$. It remains to verify the assumptions of Fatou's lemma, i.e., that the integrand at the left-hand side of (4.5) has a lower bound independent of $\delta$. When $D u_{\varepsilon}=0$, this follows directly from the inequality

$$
D^{2} u_{\varepsilon} \leq \frac{q-1}{\varepsilon}\left|D u_{\varepsilon}\right|^{\frac{q-2}{q-1}} I,
$$

which holds by Lemma 4.6. When $D u_{\varepsilon} \neq 0$, we recall that by Lipschitz continuity $\partial_{t} u_{\varepsilon}$ and $D u_{\varepsilon}$ are uniformly bounded in $\boldsymbol{\Xi}_{\varepsilon}$, and estimate

$$
\begin{aligned}
& -\left(\delta+\left|D u_{\varepsilon}\right|^{2}\right)^{\frac{p-2}{2}}\left(\Delta u_{\varepsilon}+\frac{p-2}{\delta+\left|D u_{\varepsilon}\right|^{2}} \Delta_{\infty} u_{\varepsilon}\right) \\
& =-\frac{\left(\delta+\left|D u_{\varepsilon}\right|^{2}\right)^{\frac{p-2}{2}}}{\delta+\left|D u_{\varepsilon}\right|^{2}}\left(\left|D u_{\varepsilon}\right|^{2}\left(\Delta u_{\varepsilon}+\frac{p-2}{\left|D u_{\varepsilon}\right|^{2}} \Delta_{\infty} u_{\varepsilon}\right)+\delta \Delta u_{\varepsilon}\right) \\
& \geq-\frac{\left(\delta+\left|D u_{\varepsilon}\right|^{2}\right)^{\frac{p-2}{2}}}{\delta+\left|D u_{\varepsilon}\right|^{2}} \frac{(q-1)}{\varepsilon}\left(\left|D u_{\varepsilon}\right|^{\frac{q-2}{q-1}+2}(N+p-2)+\left|D u_{\varepsilon}\right|^{\frac{q-2}{q-1}} \delta N\right) \\
& \geq-\left(\delta+\left|D u_{\varepsilon}\right|^{2}\right)^{\frac{p-2}{2}} \frac{(q-1)}{\varepsilon}\left|D u_{\varepsilon}\right|^{\frac{q-2}{q-1}}(2 N+p-2)
\end{aligned}
$$




$$
\begin{aligned}
& \geq-\left|D u_{\varepsilon}\right|^{p-2+\frac{q-2}{q-1}} \frac{(q-1)}{\varepsilon}(2 N+p-2) \\
& \geq-\left\|D u_{\varepsilon}\right\|_{L^{\infty}\left(\Xi_{\varepsilon}\right)}^{p-2+\frac{q-2}{q-1}} \frac{(q-1)}{\varepsilon}(2 N+p-2),
\end{aligned}
$$

where we used that $p-2+\frac{q-2}{q-1}>0$. Hence, the assumptions of Fatou's lemma hold.

If $u_{\varepsilon}$ is the sequence of inf-convolutions of a viscosity supersolution to (1.1), then by next Caccioppoli's inequality the sequence $D u_{\varepsilon}$ converges weakly in $L_{l o c}^{p}(\Xi)$ up to a subsequence. However, we need stronger convergence to pass to the limit under the integral sign of

$$
\int_{\Xi}-u_{\varepsilon} \partial_{t} \varphi+\left|D u_{\varepsilon}\right|^{p-2} D u_{\varepsilon} \cdot D \varphi-\varphi f\left(D u_{\varepsilon}\right) \mathrm{d} z \geq 0
$$

For this end, we show in Lemma 4.4 that $D u_{\varepsilon}$ converges in $L_{l o c}^{r}(\Xi)$ for all $1<r<p$.

Lemma 4.3. (Caccioppoli's inequality) Let $1<p<\infty$. Assume that $u$ is a locally Lipschitz continuous weak supersolution to (1.1) in $\Xi$. Then, there is a constant $C=$ $C\left(p, \beta, C_{f}\right)$ such that for any test function $\xi \in C_{0}^{\infty}(\Xi)$ we have

$$
\int_{\Xi} \xi^{p}|D u|^{p} \mathrm{~d} z \leq C \int_{\Xi} M^{2} \partial_{t} \xi^{p}+M^{p}|D \xi|^{p}+\left(M^{\frac{p}{p-\beta}}+M\right) \xi^{p} \mathrm{~d} z
$$

where $M=\|u\|_{L^{\infty}(\mathrm{spt} \xi)}$.

Proof. Since $u$ is locally Lipschitz continuous, the function $\varphi:=(M-u) \xi^{p}$ is an admissible test function. Testing the weak formulation of (1.1) with $\varphi$ yields

$$
\int_{\Xi} \xi^{p}|D u|^{p} \mathrm{~d} z \leq \int_{\Xi} u \partial_{t} \varphi+p \xi^{p-1}(M-u)|D u|^{p-1}|D \xi|+\varphi f(D u) \mathrm{d} z .
$$

We have by integration by parts

$$
\begin{aligned}
\int_{\Xi} u \partial_{t} \varphi \mathrm{d} z & =\int_{\Xi}-\xi^{p} u \partial_{t} u+u(M-u) \partial_{t} \xi^{p} \mathrm{~d} z \\
& =\int_{\Xi}-\frac{1}{2} \xi^{p} \partial_{t} u^{2}+u(M-u) \partial_{t} \xi^{p} \mathrm{~d} z \\
& =\int_{\Xi} \frac{1}{2} u^{2} \partial_{t} \xi^{p}+u(M-u) \partial_{t} \xi^{p} \mathrm{~d} z \leq \int_{\Xi} C M^{2} \partial_{t} \xi^{p} \mathrm{~d} z
\end{aligned}
$$

By Young's inequality,

$$
\int_{\Xi} p \xi^{p-1}(M-u)|D u|^{p-1}|D \xi| \mathrm{d} z \leq \int_{\Xi} \frac{1}{4} \xi^{p}|D u|^{p} \mathrm{~d} z+C(p) \int_{\Xi} M^{p}|D \xi|^{p} \mathrm{~d} z
$$


Using the growth condition (G1) and Young's inequality, we get

$$
\begin{aligned}
& \int_{\Xi} \varphi f(D u) \mathrm{d} z \\
& \quad \leq \int_{\Xi}(M-u) \xi^{p} C_{f}\left(1+|D u|^{\beta}\right) \mathrm{d} z \\
& \quad=\int_{\Xi} C_{f}(M-u) \xi^{p-\beta} \xi^{\beta}|D u|^{\beta}+C_{f}(M-u) \xi^{p} \mathrm{~d} z \\
& \quad \leq \int_{\Xi} \frac{1}{4} \xi^{p}|D u|^{p}+C\left(p, \beta, C_{f}\right)(M-u)^{\frac{p}{p-\beta}} \xi^{p}+C_{f}(M-u) \xi^{p} \mathrm{~d} z \\
& \quad \leq \int_{\Xi} \frac{1}{4} \xi^{p}|D u|^{p}+C\left(p, \beta, C_{f}\right)\left(M^{\frac{p}{p-\beta}}+M\right) \xi^{p} \mathrm{~d} z .
\end{aligned}
$$

Combining these estimates with (4.7) and absorbing the terms with $D u$ to the left-hand side yields the desired inequality.

The proof of Lemma 4.4 is based on that of Lemma 5 in [20], see also Theorem 5.3 in [15]. For the convenience of the reader, we give the full details.

Lemma 4.4. Let $1<p<\infty$. Suppose that $\left(u_{j}\right)$ is a sequence of locally Lipschitz continuous weak supersolutions to (1.1) such that $u_{j} \rightarrow u$ in $L_{l o c}^{p}(\Xi)$. Then, $\left(D u_{j}\right)$ is a Cauchy sequence in $L_{l o c}^{r}(\Xi)$ for any $1<r<p$.

Proof. Let $U \Subset \Xi$ and take a cutoff function $\theta \in C_{0}^{\infty}(\Xi)$ such that $0 \leq \theta \leq 1$ and $\theta \equiv 1$ in $U$. For $\delta>0$, we set

$$
w_{j k}= \begin{cases}\delta, & u_{j}-u_{k}>\delta, \\ u_{j}-u_{k}, & \left|u_{j}-u_{k}\right| \leq \delta, \\ -\delta, & u_{j}-u_{k}<-\delta .\end{cases}
$$

Then, the function $\left(\delta-w_{j k}\right) \theta$ is an admissible test function with a time derivative since it is Lipschitz continuous. Since $u_{j}$ is a weak supersolution, testing the weak formulation of (1.1) with $\left(\delta-w_{j k}\right) \theta$ yields

$$
\begin{aligned}
0 \leq & \int_{\Xi}-u_{j} \partial_{t}\left(\left(\delta-w_{j k}\right) \theta\right)+\left|D u_{j}\right|^{p-2} D u_{j} \cdot D\left(\left(\delta-w_{j k}\right) \theta\right)-\left(\delta-w_{j k}\right) \theta f\left(D u_{j}\right) \mathrm{d} z \\
= & \int_{\Xi}-\theta\left|D u_{j}\right|^{p-2} D u_{j} \cdot D w_{j k}+\left(\delta-w_{j k}\right)\left|D u_{j}\right|^{p-2} D u_{j} \cdot D \theta-\left(\delta-w_{j k}\right) \theta f\left(D u_{j}\right) \\
& +u_{j} \partial_{t}\left(w_{j k}\right) \theta-\left(\delta-w_{j k}\right) u_{j} \partial_{t} \theta \mathrm{d} z .
\end{aligned}
$$

Since $\left|w_{j k}\right| \leq \delta$ and $D w_{j k}=\chi_{\left\{\left|u_{j}-u_{k}\right|<\delta\right\}}\left(D u_{j}-D u_{k}\right)$, the above becomes

$$
\begin{aligned}
& \int_{\left\{\left|u_{j}-u_{k}\right|<\delta\right\}} \theta\left|D u_{j}\right|^{p-2} D u_{j} \cdot\left(D u_{j}-D u_{k}\right) \mathrm{d} z \\
& \quad \leq \int_{\Xi} 2 \delta\left|D u_{j}\right|^{p-1}|D \theta|+2 \delta \theta\left|f\left(D u_{j}\right)\right|+u_{j} \partial_{t}\left(w_{j k}\right) \theta+2 \delta\left|u_{j}\right|\left|\partial_{t} \theta\right| \mathrm{d} z .
\end{aligned}
$$


Since $u_{k}$ is a weak supersolution, the same arguments as above but testing this time with $\left(\delta+w_{j k}\right) \theta$ yield the analogous estimate

$$
\begin{aligned}
& \int_{\left\{\left|u_{j}-u_{k}\right|<\delta\right\}}-\theta\left|D u_{k}\right|^{p-2} D u_{k} \cdot\left(D u_{j}-D u_{k}\right) \mathrm{d} z \\
& \quad \leq \int_{\Xi} 2 \delta\left|D u_{k}\right|^{p-1}|D \theta|+2 \delta \theta\left|f\left(D u_{k}\right)\right|-u_{k} \partial_{t}\left(w_{j k}\right) \theta+2 \delta\left|u_{k}\right|\left|\partial_{t} \theta\right| \mathrm{d} z .
\end{aligned}
$$

Summing up these two inequalities, we arrive at

$$
\begin{aligned}
& \int_{\left\{\left|u_{j}-u_{k}\right|<\delta\right\}} \theta\left(\left|D u_{j}\right|^{p-2} D u_{j}-\left|D u_{k}\right|^{p-2} D u_{k}\right) \cdot\left(D u_{j}-D u_{k}\right) \mathrm{d} z \\
& \quad \leq 2 \delta \int_{\Xi}|D \theta|\left(\left|D u_{j}\right|^{p-1}+\left|D u_{k}\right|^{p-1}\right) \mathrm{d} z+2 \delta \int_{\Xi} \theta\left(\left|f\left(D u_{j}\right)\right|+\left|f\left(D u_{k}\right)\right|\right) \mathrm{d} z \\
& \quad+\int_{\Xi}\left(u_{j}-u_{k}\right) \partial_{t}\left(w_{j k}\right) \theta \mathrm{d} z+2 \delta \int_{\Xi}\left(\left|u_{j}\right|+\left|u_{k}\right|\right)\left|\partial_{t} \theta\right| \mathrm{d} z \\
& =: I_{1}+I_{2}+I_{3}+I_{4} .
\end{aligned}
$$

We proceed to estimate these integrals. Denoting $M:=\sup _{j}\left\|u_{j}\right\|_{L^{\infty}(\operatorname{spt} \theta)}<\infty$, we have by the Caccioppoli's inequality Lemma 4.3

$$
\sup _{j} \int_{\operatorname{spt} \theta}\left|D u_{j}\right|^{p} \mathrm{~d} z \leq C\left(p, \beta, C_{f}, \theta, M\right) .
$$

The estimate (4.9) and Hölder's inequality imply that

$$
I_{1} \leq \delta C\left(p, \beta, C_{f}, \theta, M\right) .
$$

To estimate $I_{2}$, we also use the growth condition $(\mathrm{G} 1)$ and the assumption $\beta<p$. We get

$$
I_{2} \leq 2 \delta \int_{\Xi} \theta C_{f}\left(2+\left|D u_{j}\right|^{\beta}+\left|D u_{k}\right|^{\beta}\right) \mathrm{d} z \leq \delta C\left(p, \beta, C_{f}, \theta, M\right) .
$$

The integral $I_{3}$ is estimated using integration by parts and that $\left|w_{j k}\right| \leq \delta$

$I_{3}=\int_{\Xi} \theta\left(u_{j}-u_{k}\right) \partial_{t}\left(w_{j k}\right) \mathrm{d} z=\int_{\Xi} \frac{1}{2} \theta \partial_{t} w_{j k}^{2} \mathrm{~d} z=\int_{\Xi}-\frac{1}{2} w_{j k}^{2} \partial_{t} \theta \mathrm{d} z \leq \delta C(\theta, M)$.

For the last integral, we have directly $I_{4} \leq \delta C(\theta, M)$. Combining these estimates with (4.8), we arrive at

$$
\int_{\left\{\left|u_{j}-u_{k}\right|<\delta\right\}} \theta\left(\left|D u_{j}\right|^{p-2} D u_{j}-\left|D u_{k}\right|^{p-2} D u_{k}\right) \cdot\left(D u_{j}-D u_{k}\right) \mathrm{d} z \leq \delta C_{0},
$$

where $C_{0}=C\left(p, \beta, C_{f}, \theta, M\right)$. If $1<p<2$, Hölder's inequality and the algebraic inequality (3.8) give the estimate (recall that $1<r<p$ and $\theta \equiv 1$ in $U$ )

$$
\int_{U \cap\left\{\left|u_{j}-u_{k}\right|<\delta\right\}}\left|D u_{j}-D u_{k}\right|^{r} \mathrm{~d} z
$$




$$
\begin{aligned}
\leq & \left(\int_{U \cap\left\{\left|u_{j}-u_{k}\right|<\delta\right\}}\left(1+\left|D u_{j}\right|^{2}+\left|D u_{k}\right|^{2}\right)^{\frac{r(2-p)}{2(2-r)}} \mathrm{d} z\right)^{\frac{2-r}{2}} \\
& \cdot\left(\int_{U \cap\left\{\left|u_{j}-u_{k}\right|<\delta\right\}} \frac{\left|D u_{j}-D u_{k}\right|^{2}}{\left(1+\left|D u_{j}\right|^{2}+\left|D u_{k}\right|^{2}\right)^{\frac{2-p}{2}}} \mathrm{~d} z\right)^{\frac{r}{2}} \\
\leq & C\left(p, \beta, r, C_{f}, \theta, M\right) \\
& \cdot\left(\int_{\left\{\left|u_{j}-u_{k}\right|<\delta\right\}} \theta\left(\left|D u_{j}\right|^{p-2} D u_{j}-\left|D u_{k}\right|^{p-2} D u_{k}\right) \cdot\left(D u_{j}-D u_{k}\right) \mathrm{d} z\right)^{\frac{r}{2}},
\end{aligned}
$$

where in the last inequality we also used (4.9) with the knowledge $\frac{r(2-p)}{(2-r)} \leq \frac{p(2-p)}{2-p}=$ $p$.

If $p \geq 2$, Hölder's inequality and the algebraic inequality (3.12) imply

$$
\begin{aligned}
& \int_{U \cap\left\{\left|u_{j}-u_{k}\right|<\delta\right\}}\left|D u_{j}-D u_{k}\right|^{r} \mathrm{~d} z \\
& \quad \leq\left(\int_{\Xi} 1 \mathrm{~d} z\right)^{\frac{p-r}{p}}\left(\int_{U \cap\left\{\left|u_{j}-u_{k}\right|<\delta\right\}}\left|D u_{j}-D u_{k}\right|^{p} \mathrm{~d} z\right)^{\frac{r}{p}} \\
& \quad \leq C(p, r)\left(\int_{\left\{\left|u_{j}-u_{k}\right|<\delta\right\}} \theta\left(\left|D u_{j}\right|^{p-2} D u_{j}-\left|D u_{k}\right|^{p-2} D u_{k}\right) \cdot\left(D u_{j}-D u_{k}\right) \mathrm{d} z\right)^{\frac{r}{p}} .
\end{aligned}
$$

Hence, (4.10) leads to

$$
\int_{U \cap\left\{\left|u_{j}-u_{k}\right|<\delta\right\}}\left|D u_{j}-D u_{k}\right|^{r} \mathrm{~d} z \leq \delta^{\frac{r}{\max (2, p)}} C\left(p, \beta, r, C_{f}, \theta, M\right) .
$$

On the other hand, Hölder's and Tchebysheff's inequalities with (4.9) imply

$$
\begin{aligned}
& \int_{U \cap\left\{\left|u_{j}-u_{k}\right| \geq \delta\right\}}\left|D u_{j}-D u_{k}\right|^{r} \mathrm{~d} z \\
& \quad \leq\left|U \cap\left\{\left|u_{j}-u_{k}\right| \geq \delta\right\}\right|^{\frac{p-r}{p}}\left(\int_{U \cap\left\{\left|u_{j}-u_{k}\right| \geq \delta\right\}}\left|D u_{j}-D u_{k}\right|^{p} \mathrm{~d} z\right)^{\frac{r}{p}} \\
& \quad \leq \delta^{r-p}\left\|u_{j}-u_{k}\right\|_{L^{p}(U)}^{p-r} C\left(p, \beta, r, C_{f}, \theta, M\right) .
\end{aligned}
$$

So we arrive at

$$
\int_{U}\left|D u_{j}-D u_{k}\right|^{r} \mathrm{~d} z \leq\left(\delta^{\frac{r}{\max (2, p)}}+\delta^{r-p}\left\|u_{j}-u_{k}\right\|_{L^{p}(U)}^{p-r}\right) C\left(p, \beta, r, C_{f}, \theta, M\right) .
$$

Taking first small $\delta>0$ and then large $j, k$, we can make the right-hand side arbitrarily small.

Now we are ready to prove the main result of this section which states that bounded viscosity supersolutions are weak supersolutions.

Theorem 4.5. Let $1<p<\infty$. Let $u$ be a bounded viscosity supersolution to (1.1) in $\Xi$. Then, $u$ is a weak supersolution to (1.1) in $\Xi$. 
Proof. Fix a non-negative test function $\varphi \in C_{0}^{\infty}(\Xi)$ and take an open cylinder $\Omega_{t_{1}, t_{2}} \Subset \Xi$ such that spt $\varphi \Subset \Omega_{t_{1}, t_{2}}$. Let $\varepsilon>0$ be so small that $\Omega_{t_{1}, t_{2}} \Subset \Xi_{\varepsilon}$. Then, Lemma 4.2 implies that $u_{\varepsilon}$ is a weak supersolution to (1.1) in $\Xi_{\varepsilon}$. Therefore, by the Caccioppoli's inequality Lemma $4.3, D u_{\varepsilon}$ is bounded in $L^{p}\left(\Omega_{t_{1}, t_{2}}\right)$. Hence, $D u_{\varepsilon}$ converges weakly in $L^{p}\left(\Omega_{t_{1}, t_{2}}\right)$ up to a subsequence. Since also $u_{\varepsilon} \rightarrow u$ in $L^{p}\left(\Omega_{t_{1}, t_{2}}\right)$ by dominated convergence and the fact that $u_{\varepsilon} \rightarrow u$ pointwise in $\Omega_{t_{1}, t_{2}}$, it follows that $u \in L^{p}\left(t_{1}, t_{2} ; W^{1, p}(\Omega)\right)$.

Since $u_{\varepsilon}$ is a weak supersolution, it remains to show that up to a subsequence

$$
\lim _{\varepsilon \rightarrow 0} \int_{\Omega_{t_{1}, t_{2}}} u_{\varepsilon} \partial_{t} \varphi+\left|D u_{\varepsilon}\right|^{p-2} D u_{\varepsilon} \cdot D \varphi \mathrm{d} z=\int_{\Omega_{t_{1}, t_{2}}} u \partial_{t} \varphi+|D u|^{p-2} D u \cdot D \varphi \mathrm{d} z
$$

and

$$
\lim _{\varepsilon \rightarrow 0} \int_{\Omega_{t_{1}, t_{2}}} \varphi f\left(D u_{\varepsilon}\right) \mathrm{d} z=\int_{\Omega_{t_{1}, t_{2}}} \varphi f(D u) \mathrm{d} z .
$$

Since $u_{\varepsilon} \rightarrow u$ in $L^{p}\left(\Omega_{t_{1}, t_{2}}\right)$ and $D u_{\varepsilon} \rightarrow D u$ in $L^{r}\left(\Omega_{t_{1}, t_{2}}\right)$ for any $1<r<p$ by Lemma 4.4, the claim (4.11) follows by applying the vector inequality (see [19, pp. 95-96])

$$
\left.|| a\right|^{p-2} a-|b|^{p-2} b \mid \leq \begin{cases}2^{2-p}|a-b|^{p-1} & \text { when } p<2 \\ 2^{-1}\left(|a|^{p-2}+|b|^{p-2}\right)|a-b| & \text { when } p \geq 2 .\end{cases}
$$

To show (4.12), let $M \geq 1$ and write using the growth condition (G1)

$$
\begin{aligned}
& \int_{\Omega_{t_{1}, t_{2}}}\left|f\left(D u_{\varepsilon}\right)-f(D u)\right| \mathrm{d} z \\
& \quad \leq \int_{\left\{\left|D u_{\varepsilon}\right|<M\right\}}\left|f\left(D u_{\varepsilon}\right)-f(D u)\right| \mathrm{d} z+\int_{\left\{\left|D u_{\varepsilon}\right| \geq M\right\}} C_{f}\left(2+\left|D u_{\varepsilon}\right|^{\beta}+|D u|^{\beta}\right) \mathrm{d} z \\
& \quad=: I_{1}+I_{2} .
\end{aligned}
$$

Then, by Hölder's inequality

$$
\begin{aligned}
I_{2} & =C_{f} \int_{\left\{\left|D u_{\varepsilon}\right| \geq M\right\}} \frac{2\left|D u_{\varepsilon}\right|^{p}}{\left|D u_{\varepsilon}\right|^{p}}+\frac{\left|D u_{\varepsilon}\right|^{p}}{\left|D u_{\varepsilon}\right|^{p-\beta}}+\frac{|D u|^{\beta}\left|D u_{\varepsilon}\right|^{p-\beta}}{\left|D u_{\varepsilon}\right|^{p-\beta}} \mathrm{d} z \\
& \leq C_{f}\left(\frac{2}{M^{p}}+\frac{1}{M^{p-\beta}}\right)\left\|D u_{\varepsilon}\right\|_{L^{p}\left(\Omega_{t_{1}, t_{2}}\right)}^{p}+C_{f} \frac{1}{M^{p-\beta}}\|D u\|_{L^{p}\left(\Omega_{t_{1}, t_{2}}\right)}^{\beta}\left\|D u_{\varepsilon}\right\|_{L^{p}\left(\Omega_{t_{1}, t_{2}}\right)}^{p-\beta} \\
& \leq \frac{1}{M^{p-\beta}} C\left(p, \beta, C_{f},\|D u\|_{L^{p}\left(\Omega_{t_{1}, t_{2}}\right)}, \sup _{\varepsilon}\left\|D u_{\varepsilon}\right\|_{L^{p}\left(\Omega_{t_{1}, t_{2}}\right)}\right) .
\end{aligned}
$$

On the other hand, we have $\left|f\left(D u_{\varepsilon}\right)-f(D u)\right| \rightarrow 0$ a.e. in $\Omega_{t_{1}, t_{2}}$ up to a subsequence and the integrand in $I_{1}$ is dominated by an integrable function since the growth condition (G1) implies

$$
\left|f\left(D u_{\varepsilon}\right)-f(D u)\right| \leq C_{f}\left(2+|M|^{\beta}+|D u|^{\beta}\right) \text { when }\left|D u_{\varepsilon}\right|<M .
$$


Hence, for any $M \geq 1$, we have $I_{1} \rightarrow 0$ as $\varepsilon \rightarrow 0$ by the dominated convergence theorem. By taking first large $M \geq 1$ and then small $\varepsilon>0$, we can make $I_{1}+I_{2}$ arbitrarily small.

The rest of this section is devoted to the properties of the inf-convolution. The facts in the following lemma are well known, see, e.g., [6,11,14] or [24].

Lemma 4.6. Assume that $u: \Xi \rightarrow \mathbb{R}$ is lower semicontinuous and bounded. Then, $u_{\varepsilon}$ has the following properties.

(i) We have $u_{\varepsilon} \leq u$ in $\Xi$ and $u_{\varepsilon} \rightarrow$ upointwise as $\varepsilon \rightarrow 0$.

(ii) Denote $r(\varepsilon):=\left(q \varepsilon^{q-1} \operatorname{osc}_{\Xi} u\right)^{\frac{1}{q}}, t(\varepsilon):=\left(2 \varepsilon \operatorname{osc}_{\Xi} u\right)^{\frac{1}{2}}$. For $(x, t) \in \mathbb{R}^{N+1}$, set

$$
\Xi_{\varepsilon}:=\left\{(x, t) \in \Xi: B_{r(\varepsilon)}(x) \times(t-t(\varepsilon), t+t(\varepsilon)) \Subset \Xi\right\} .
$$

Then, for any $(x, t) \in \Xi_{\varepsilon}$ there exists $\left(x_{\varepsilon}, t_{\varepsilon}\right) \in \bar{B}_{r(\varepsilon)}(x) \times[t-t(\varepsilon), t+t(\varepsilon)]$ such that

$$
u_{\varepsilon}(x, t)=u\left(x_{\varepsilon}, t_{\varepsilon}\right)+\frac{\left|x-x_{\varepsilon}\right|^{q}}{q \varepsilon^{q-1}}+\frac{\left|t-t_{\varepsilon}\right|^{2}}{2 \varepsilon} .
$$

(iii) The function $u_{\varepsilon}$ is semi-concave in $\Xi_{\varepsilon}$ with a semi-concavity constant depending only on $u, q$ and $\varepsilon$.

(iv) Assume that $u_{\varepsilon}$ is differentiable in time and twice differentiable in space at $(x, t) \in \Xi_{\varepsilon}$. Then,

$$
\begin{aligned}
\partial_{t} u_{\varepsilon}(x, t) & =\frac{t-t_{\varepsilon}}{\varepsilon}, \\
D u_{\varepsilon}(x, t) & =\left(x-x_{\varepsilon}\right) \frac{\left|x-x_{\varepsilon}\right|^{q-2}}{\varepsilon^{q-1}}, \\
D^{2} u_{\varepsilon}(x, t) & \leq \frac{q-1}{\varepsilon}\left|D u_{\varepsilon}\right|^{\frac{q-2}{q-1}} I .
\end{aligned}
$$

Next, we show that the inf-convolution of a viscosity supersolution to (1.1) is still a supersolution in the smaller set $\boldsymbol{\Xi}_{\varepsilon}$. Since the inf-convolution is "flat enough," that is, since $q>p /(p-1)$, the inf-convolution essentially cancels the singularity of the $p$-Laplace operator. This allows us to extract information on the time derivative at those points of differentiability where $D u_{\varepsilon}$ vanishes.

Lemma 4.7. Let $1<p<\infty$. Let $u$ be a bounded viscosity supersolution to (1.1) in $\Xi$. Then, the inf-convolution $u_{\varepsilon}$ is also a viscosity supersolution to (1.1) in $\Xi_{\varepsilon}$.

Moreover, if $u_{\varepsilon}$ is differentiable in time and twice differentiable in space at $(x, t) \in$ $\Xi_{\varepsilon}$ and $D u_{\varepsilon}(x, t)=0$, then $\partial_{t} u_{\varepsilon}(x, t)-f(0) \geq 0$.

Proof. Assume that $\varphi$ touches $u_{\varepsilon}$ from below at $(x, t) \in \Xi_{\varepsilon}$. Let $\left(x_{\varepsilon}, t_{\varepsilon}\right)$ be like in the property (ii) of Lemma 4.6. Then,

$$
\varphi(x, t)=u_{\varepsilon}(x, t)=u\left(x_{\varepsilon}, t_{\varepsilon}\right)+\frac{\left|x-x_{\varepsilon}\right|^{q}}{q \varepsilon^{q-1}}+\frac{\left|t-t_{\varepsilon}\right|^{2}}{2 \varepsilon},
$$




$$
\varphi(y, \tau) \leq u_{\varepsilon}(y, \tau) \leq u(z, s)+\frac{|y-z|^{q}}{q \varepsilon^{q-1}}+\frac{|\tau-s|^{2}}{2 \varepsilon} \text { for all }(y, \tau),(z, s) \in \Xi .
$$

Set

$$
\psi(z, s):=\varphi\left(z+x-x_{\varepsilon}, s+t-t_{\varepsilon}\right)-\frac{\left|x-x_{\varepsilon}\right|^{q}}{q \varepsilon^{q-1}}-\frac{\left|t-t_{\varepsilon}\right|^{2}}{2 \varepsilon} .
$$

Then, $\psi$ touches $u$ from below at $\left(x_{\varepsilon}, t_{\varepsilon}\right)$ since by (4.13)

$$
\psi\left(x_{\varepsilon}, t_{\varepsilon}\right)=\varphi(x, t)-\frac{\left|x-x_{\varepsilon}\right|^{q}}{q \varepsilon^{q-1}}-\frac{\left|t-t_{\varepsilon}\right|^{2}}{2 \varepsilon}=u\left(x_{\varepsilon}, t_{\varepsilon}\right)
$$

and selecting $(y, \tau)=\left(z+x-x_{\varepsilon}, s+t-t_{\varepsilon}\right)$ in (4.14) gives

$$
\psi(z, s)=\varphi\left(z+x-x_{\varepsilon}, s+t-t_{\varepsilon}\right)-\frac{\left|x-x_{\varepsilon}\right|^{q}}{q \varepsilon^{q-1}}-\frac{\left|t-t_{\varepsilon}\right|^{2}}{2 \varepsilon} \leq u(z, s) .
$$

Since $u$ is a viscosity supersolution, it follows that

$$
\begin{aligned}
0 \leq & \limsup _{\substack{(z, s) \rightarrow\left(x_{\varepsilon}, t_{\varepsilon}\right) \\
z \neq x_{\varepsilon}}}\left(\partial_{s} \psi(z, s)-\Delta_{p} \psi(z, s)-f(D \psi(z, s))\right) \\
& =\lim _{\substack{(z, s) \rightarrow(x, t) \\
z \neq x}}\left(\partial_{s} \varphi(z, s)-\Delta_{p} \varphi(z, s)-f(D \varphi(z, s))\right),
\end{aligned}
$$

and the first claim is proven. To prove the second claim, assume that $u_{\varepsilon}$ is differentiable in time and twice differentiable in space at $(x, t) \in \Xi_{\varepsilon}$ and $D u_{\varepsilon}(x, t)=0$. By the property (iv) in Lemma 4.6, we have $x=x_{\varepsilon}$, so that

$$
u_{\varepsilon}(x, t)=u\left(x, t_{\varepsilon}\right)+\frac{\left|t-t_{\varepsilon}\right|^{2}}{2 \varepsilon} .
$$

Hence, by the definition of inf-convolution $u(y, s)+\frac{|x-y|^{q}}{q \varepsilon^{q-1}}+\frac{|t-s|^{2}}{2 \varepsilon} \geq u_{\varepsilon}(x, t)=u\left(x, t_{\varepsilon}\right)+\frac{\left|t-t_{\varepsilon}\right|^{2}}{2 \varepsilon}$ for all $(y, s) \in \Xi$.

Arranging the terms as

$$
u(y, s) \geq u\left(x, t_{\varepsilon}\right)-\frac{|x-y|^{q}}{q \varepsilon^{q-1}}-\frac{|t-s|^{2}}{2 \varepsilon}+\frac{\left|t-t_{\varepsilon}\right|^{2}}{2 \varepsilon}=: \phi(y, s),
$$

we see that the function $\phi$ touches $u$ from below at $\left(x, t_{\varepsilon}\right)$. Since $u$ is a viscosity supersolution and $D \phi(y, s) \neq 0$ when $y \neq x$, we have

$$
\limsup _{\substack{(y, s) \rightarrow\left(x, t_{\varepsilon}\right) \\ y \neq x}}\left(\partial_{s} \phi(y, s)-\Delta_{p} \phi(y, s)-f(D \phi(y, s))\right) \geq 0 .
$$


On the other hand, since $q>p /(p-1)$, we have $\Delta_{p} \phi(y, s) \rightarrow 0$ as $y \rightarrow x$. Hence, we get

$$
0 \leq \partial_{s} \phi\left(x, t_{\varepsilon}\right)-f(0)=\frac{t-t_{\varepsilon}}{\varepsilon}-f(0)=\partial_{t} u_{\varepsilon}(x, t)-f(0),
$$

where the last equality follows from the property (iv) in Lemma 4.6.

Remark 4.8. Semi-concavity implies that the inf-convolution $u_{\varepsilon}$ is locally Lipschitz in $\Xi_{\varepsilon}$ (see [8, p. 267]). Therefore, $u_{\varepsilon}$ is differentiable almost everywhere in $\Xi_{\varepsilon}$, $\partial_{t} u_{\varepsilon} \in L_{\text {loc }}^{\infty}\left(\Xi_{\varepsilon}\right)$ and $u_{\varepsilon} \in L^{\infty}\left(t_{1}, t_{2} ; W^{1, \infty}(\Omega)\right)$ for any $\Omega_{t_{1}, t_{2}} \Subset \Xi_{\varepsilon}$ (see [8, p. 266]).

Moreover, since the function $\phi(x, t):=u_{\varepsilon}(x, t)-C(q, \varepsilon, u)\left(|x|^{2}+|t|^{2}\right)$ is concave, Alexandrov's theorem implies that $u_{\varepsilon}$ is twice differentiable almost everywhere in $\Xi_{\varepsilon}$. Furthermore, the proof of Alexandrov's theorem in [8, p. 273] establishes that if $\phi_{j}$ is the standard mollification of $\phi$, then $D^{2} \phi_{j} \rightarrow D^{2} \phi$ almost everywhere in $\Xi_{\varepsilon}$.

\section{Lower semicontinuity of supersolutions}

We show the lower semicontinuity of weak supersolutions when $p \geq 2$ and the function $f \in C\left(\mathbb{R}^{N}\right)$ satisfies that $f(0)=0$ as well as the stronger growth condition

$$
|f(\xi)| \leq C_{f}\left(1+|\xi|^{p-1}\right) .
$$

Our proof follows the method of Kuusi [17], but the first-order term causes some modifications. In particular, our essential supremum estimate is slightly different, see Theorem 5.3 and the brief discussion before it. The assumption $f(0)=0$ is used to ensure that the positive part $u_{+}$of a subsolution is still a subsolution.

We begin by proving estimates for the essential supremum of a subsolution using Moser's iteration technique. We first need the following Caccioppoli's inequalities.

Lemma 5.1. (Caccioppoli's inequalities) Assume that $p \geq 2$ and that (G2) holds. Suppose that $u$ is a non-negative weak subsolution to $(1.1)$ in $\Omega_{t_{1}, t_{2}}$ and $u \in L^{p-1+\lambda}\left(\Omega_{t_{1}, t_{2}}\right)$ for some $\lambda \geq 1$. Then, there exists a constant $C=C\left(p, C_{f}\right)$ that satisfies the estimates

$$
\begin{aligned}
& \underset{t_{1}<\tau<t_{2}}{\operatorname{ess} \sup } \int_{\Omega} u^{1+\lambda}(x, \tau) \zeta^{p}(x, \tau) \mathrm{d} x \\
& \quad \leq C \int_{\Omega_{t_{1}, t_{2}}} \lambda u^{p-1+\lambda}|D \zeta|^{p}+u^{1+\lambda}\left|\partial_{t} \zeta\right| \zeta^{p-1}+\lambda\left(u^{\lambda}+u^{p-1+\lambda}\right) \zeta^{p} \mathrm{~d} z
\end{aligned}
$$

and

$$
\begin{aligned}
& \int_{\Omega_{t_{1}, t_{2}}}\left|D\left(u^{\frac{p-1+\lambda}{p}} \zeta\right)\right|^{p} \mathrm{~d} z \\
& \quad \leq C \int_{\Omega_{t_{1}, t_{2}}} \lambda^{p} u^{p-1+\lambda}|D \zeta|^{p}+\lambda^{p-1} u^{1+\lambda}\left|\partial_{t} \zeta\right| \zeta^{p-1}+\lambda^{p}\left(u^{\lambda}+u^{p-1+\lambda}\right) \zeta^{p} \mathrm{~d} z
\end{aligned}
$$

for all non-negative $\zeta \in C^{\infty}\left(\Omega \times\left[t_{1}, t_{2}\right]\right)$ such that $\operatorname{spt} \zeta(\cdot, t) \Subset \Omega$ and $\zeta\left(x, t_{1}\right)=0$. Proof. We test the regularized equation in Lemma (3.1) with $\varphi:=\min \left(u^{\epsilon}, k\right)^{\lambda-1}$ $u^{\epsilon} \zeta^{p} \eta$, where $\eta$ is the following cutoff function 


$$
\eta(t)= \begin{cases}0, & t \in\left(t_{1}, s-h\right), \\ (t-s+h) / 2 h, & t \in[s-h, s+h], \\ 1, & t \in(s+h, \tau-h), \\ (-t+\tau+h) / 2 h, & t \in[\tau-h, \tau+h], \\ 0, & t \in\left(\tau+h, t_{2}\right),\end{cases}
$$

and $t_{1}<s<\tau<t_{2}, h>0$. We denote $g(l):=\int_{0}^{l} \min (r, k)^{\lambda-1} r \mathrm{~d} r$. Then, integration by parts and Lebesgue's differentiation theorem yield for a.e. $s, \tau \in\left(t_{1}, t_{2}\right)$

$$
\begin{aligned}
& \int_{\Omega_{t_{1}, t_{2}}} \partial_{t}\left(u^{\epsilon}\right) \min \left(u^{\epsilon}, k\right)^{\lambda-1} u^{\epsilon} \zeta^{p} \eta \mathrm{d} z \\
& =\int_{\Omega_{t_{1}, t_{2}}} \partial_{t} g\left(u^{\epsilon}\right) \zeta^{p} \eta \mathrm{d} z \\
& =\int_{\Omega_{t_{1}, t_{2}}}-\eta g\left(u^{\epsilon}\right) \partial_{t}\left(\zeta^{p}\right)-\zeta^{p} g\left(u^{\epsilon}\right) \partial_{t} \eta \mathrm{d} z \\
& \quad \underset{\epsilon \rightarrow 0, h \rightarrow 0}{\rightarrow} \int_{\Omega_{s, \tau}}-g(u) \partial_{t}\left(\zeta^{p}\right) \mathrm{d} z-\int_{\Omega} \zeta^{p}(x, s) g(u(x, s)) \mathrm{d} x \\
& \quad+\int_{\Omega} \zeta^{p}(x, \tau) g(u(x, \tau)) \mathrm{d} x .
\end{aligned}
$$

Letting $s \rightarrow t_{1}$ and observing that the other terms of (3.1) converge as well, we obtain for a.e. $\tau \in\left(t_{1}, t_{2}\right)$ that

$$
\begin{aligned}
& \int_{\Omega} g(u(x, \tau)) \zeta^{p}(x, \tau) \mathrm{d} x \\
& \quad \leq \int_{\Omega_{t_{1}, \tau}} g(u) \partial_{t}\left(\zeta^{p}\right)-|D u|^{p-2} D u \cdot D\left(u_{k}^{\lambda-1} u \zeta^{p}\right)+u_{k}^{\lambda-1} u \zeta^{p} f(D u) \mathrm{d} z
\end{aligned}
$$

where we have denoted $u_{k}:=\min (u, k)$. Since

$$
D u_{k}^{\lambda-1}=\chi_{\{u<k\}}(\lambda-1) u^{\lambda-2} D u,
$$

we have by Young's inequality

$$
\begin{aligned}
-|D u|^{p-2} D u \cdot D\left(u_{k}^{\lambda-1} u \zeta^{p}\right) \leq & -\zeta^{p}\left((\lambda-1) \chi\{u<k\} u^{\lambda-1}+u_{k}^{\lambda-1}\right)|D u|^{p} \\
& +p \zeta^{p-1} u_{k}^{\lambda-1} u|D u|^{p-1}|D \zeta| \\
\leq & -\frac{1}{2} \zeta^{p} u_{k}^{\lambda-1}|D u|^{p}+C(p) u^{p-1+\lambda}|D \zeta|^{p} .
\end{aligned}
$$

Moreover, by the growth condition (G2) and Young's inequality

$$
\begin{aligned}
u_{k}^{\lambda-1} u \zeta^{p} f(D u) & \leq C_{f} \zeta^{p} u_{k}^{\lambda-1} u+C_{f} \zeta^{p} u_{k}^{\lambda-1} u|D u|^{p-1} \\
& \leq C_{f} \zeta^{p} u^{\lambda-1}+C\left(p, C_{f}\right) \zeta^{p} u^{p-1+\lambda}+\frac{1}{4} \zeta^{p} u_{k}^{\lambda-1}|D u|^{p}
\end{aligned}
$$


Collecting the estimates, moving the terms with $D u$ to the left-hand side and letting $k \rightarrow \infty$, we arrive at

$$
\begin{aligned}
& \lambda^{-1} \int_{\Omega} u^{\lambda+1} \zeta^{p}(x, \tau) \mathrm{d} x+\int_{\Omega_{t_{1}, \tau}} \frac{1}{4} \zeta^{p} u^{\lambda-1}|D u|^{p} \mathrm{~d} z \\
& \quad \leq C\left(p, C_{f}\right) \int_{\Omega_{t_{1}, \tau}} \lambda^{-1} u^{\lambda+1}\left|\partial_{t} \zeta^{p}\right|+u^{p-1+\lambda}|D \zeta|^{p}+\zeta^{p}\left(u^{\lambda-1}+u^{p-1+\lambda}\right) \mathrm{d} z .
\end{aligned}
$$

Since the integrals are positive, this yields the first inequality of the lemma by taking essential supremum over $\tau$. The second inequality follows from (5.1) by using that

$$
\int_{\Omega_{t_{1}, t_{2}}}\left|D\left(u^{\frac{p-1+\lambda}{p}} \zeta\right)\right|^{p} \mathrm{~d} z \leq C(p) \int_{\Omega_{t_{1}, t_{2}}} u^{p-1+\lambda}|D \zeta|^{p}+\lambda^{p} \zeta^{p} u^{\lambda-1}|D u|^{p} \mathrm{~d} z .
$$

We first prove the following essential supremum estimate where we assume that the subsolution is bounded away from zero.

Lemma 5.2. Assume that $p \geq 2$ and that (G2) holds. Suppose that $u$ is a weak subsolution to (1.1) in $\Xi$ and $B_{R}\left(x_{0}\right) \times\left(t_{0}-T, t_{0}\right) \Subset \Xi$ where $R, T<1$ are such that

$$
\frac{R^{p}}{T} \leq 1 \text { and } u \geq\left(\frac{R^{p}}{T}\right)^{\frac{1}{p-1}}
$$

Then, there exists a constant $C\left(N, p, C_{f}\right)$ such that

$$
\operatorname{ess}_{B_{\sigma R\left(x_{0}\right) \times\left(t_{0}-\sigma^{p} T, t_{0}\right)}} u \leq C\left(\frac{T}{R^{p}}(1-\sigma)^{-N-p} \int_{B_{R}\left(x_{0}\right) \times\left(t_{0}-T, t_{0}\right)} u^{p-2+\delta} \mathrm{d} z\right)^{1 / \delta}
$$

for every $1 / 2 \leq \sigma<1$ and $1<\delta<2$.

Proof. Let $\sigma R \leq s<S<R$. For $j \in 0,1,2, \ldots$, we set

$$
R_{j}:=S-(S-s)\left(1-2^{-j}\right)
$$

and

$$
U_{j}:=B_{j} \times \Gamma_{j}:=B_{R_{j}}\left(x_{0}\right) \times\left(t_{0}-\left(R_{j} / S\right)^{p} T, t_{0}\right) .
$$

We choose test functions $\varphi_{j} \in C^{\infty}\left(\overline{U_{j}}\right)$ such that $\operatorname{spt} \varphi_{j}(\cdot, t) \Subset B_{R_{j}}\left(x_{0}\right)$,

$$
0 \leq \varphi_{j} \leq 1, \varphi_{j} \equiv 0 \text { on } \partial_{p} U_{j}, \varphi_{j} \equiv 1 \text { in } U_{j+1}
$$

and

$$
\left|D \varphi_{j}\right| \leq \frac{C}{S-s} 2^{j}, \quad\left|\partial_{t} \varphi_{j}\right| \leq \frac{R^{p}}{T} \frac{C}{(S-s)^{p}} 2^{j p} .
$$


We set $\gamma:=1+p / N$ and

$$
\lambda_{j}:=2 \gamma^{j}-1, j=0,1,2, \ldots
$$

Assuming that we already know that $u \in L^{p-1+\lambda_{j}}\left(U_{j}\right)$, then we have by a parabolic Sobolev's inequality (see [7, p7])

$$
\begin{aligned}
& \int_{U_{j+1}} u^{\kappa \alpha} \mathrm{d} z \leq \int_{U_{j}}\left(u^{\alpha / p} \varphi_{j}^{\beta / p}\right)^{\kappa p} \mathrm{~d} z \\
& \quad \leq C(N, p) \int_{U_{j}}\left|D\left(u^{\alpha / p} \varphi_{j}^{\beta / p}\right)\right|^{p} \mathrm{~d} z\left(\underset{\Gamma_{j}}{\operatorname{ess} \sup } \int_{B_{j}}\left(u^{\alpha / p} \varphi_{j}^{\beta / p}\right)^{(\kappa-1) N} \mathrm{~d} x\right)^{p / N},
\end{aligned}
$$

where

$$
\alpha=p-1+\lambda_{j}, \kappa=1+\frac{p\left(1+\lambda_{j}\right)}{N\left(p-1+\lambda_{j}\right)}, \beta=\frac{p\left(p-1+\lambda_{j}\right)}{1+\lambda_{j}} .
$$

The first estimate in Lemma 5.1 gives

$$
\begin{aligned}
& \underset{\Gamma_{j}}{\operatorname{ess} \sup } \int_{B_{j}}\left(u^{\alpha / p} \varphi_{j}^{\beta / p}\right)^{(\kappa-1) N} \mathrm{~d} x=\underset{\Gamma_{j}}{\operatorname{ess} \sup } \int_{B_{j}} u^{1+\lambda_{j}} \varphi_{j}^{p} \mathrm{~d} x \\
& \quad \leq C \lambda_{j} \int_{U_{j}} u^{p-1+\lambda_{j}}\left|D \varphi_{j}\right|^{p}+u^{1+\lambda_{j}}\left|\partial_{t} \varphi_{j}\right| \varphi_{j}^{p-1}+\left(u^{\lambda_{j}}+u^{p-1+\lambda_{j}}\right) \varphi_{j}^{p} \mathrm{~d} z .
\end{aligned}
$$

Using the second estimate with $\zeta=\varphi_{j}^{\beta / p}$, we obtain

$$
\begin{aligned}
& \int_{U_{j}}\left|D\left(u^{\alpha / p} \varphi_{j}^{\beta / p}\right)\right|^{p} \mathrm{~d} z \\
& \quad \leq C \lambda_{j}^{p} \int_{U_{j}} u^{p-1+\lambda_{j}}\left|D \varphi_{j}\right|^{p}+u^{1+\lambda_{j}}\left|\partial_{t} \varphi_{j}\right| \varphi_{j}^{p-1}+\left(u^{\lambda_{j}}+u^{p-1+\lambda_{j}}\right) \varphi_{j}^{p} \mathrm{~d} z .
\end{aligned}
$$

Combining (5.3) with (5.4) and (5.5), we arrive at

$$
\left(\int_{U_{j+1}} u^{\kappa \alpha} \mathrm{d} z\right)^{\frac{1}{\gamma}} \leq C \lambda_{j}^{p} \int_{U_{j}} \frac{2^{j p}}{(S-s)^{p}} u^{p-1+\lambda_{j}}+\frac{R^{p} 2^{j p}}{T(S-s)^{p}} u^{1+\lambda_{j}}+u^{\lambda_{j}} \mathrm{~d} z,
$$

where $\gamma=1+p / N$. We wish to iterate this inequality, but having multiple terms at the right-hand side is a problem. This is where the assumption (5.2) comes into play. Since $u \geq\left(R^{p} / T\right)^{1 /(p-1)}$, we have

$$
u^{\lambda_{j}}=\left(\frac{1}{u}\right)^{p-1} u^{p-1+\lambda_{j}} \leq\left(\frac{T}{R^{p}}\right)^{\frac{p-1}{p-1}} u^{p-1+\lambda_{j}} \leq \frac{1}{(S-s)^{p}} u^{p-1+\lambda_{j}}
$$


and since $T / R^{p} \geq 1$, we have also

$$
u^{1+\lambda_{j}}=\left(\frac{1}{u}\right)^{p-2} u^{p-1+\lambda_{j}} \leq\left(\frac{T}{R^{p}}\right)^{\frac{p-2}{p-1}} u^{p-1+\lambda_{j}} \leq \frac{T}{R^{p}} u^{p-1+\lambda_{j}} .
$$

Using these estimates it follows from (5.6) that

$$
\left(\int_{U_{j+1}} u^{\kappa \alpha} \mathrm{d} z\right)^{\frac{1}{\gamma}} \leq \frac{C \lambda_{j}^{p} 2^{j p}}{(S-s)^{p}} \int_{U_{j}} u^{p-1+\lambda_{j}} \mathrm{~d} z
$$

Observe that

$$
\kappa \alpha=p-1+\lambda_{j}(1+p / N)+p / N=p-1+\lambda_{j+1} .
$$

Hence, by denoting $Y:=C(S-s)^{-p}$, the inequality (5.7) becomes

$$
\left(\int_{U_{j+1}} u^{p-1+\lambda_{j+1}} \mathrm{~d} z\right)^{\frac{1}{\gamma}} \leq Y(2 \gamma)^{j p} \int_{U_{j}} u^{p-1+\lambda_{j}} \mathrm{~d} z
$$

We iterate this inequality. When $j=0$, it reads as

$$
\left(\int_{U_{1}} u^{p-1+\lambda_{1}} \mathrm{~d} z\right)^{\frac{1}{\gamma}} \leq Y \int_{U_{0}} u^{p} \mathrm{~d} z
$$

Then, when $j=1$, we have

$$
\left(\int_{U_{2}} u^{p-1+\lambda_{2}} \mathrm{~d} z\right)^{\frac{1}{\gamma^{2}}} \leq Y^{\frac{1}{\gamma}}(2 \gamma)^{p \frac{1}{\gamma}}\left(\int_{U_{1}} u^{p-1+\lambda_{1}} \mathrm{~d} z\right)^{\frac{1}{\gamma}} \leq Y^{1+\frac{1}{\gamma}}(2 \gamma)^{p \frac{1}{\gamma}} \int_{U_{0}} u^{p} \mathrm{~d} z .
$$

Continuing this way, we arrive at

$$
\begin{aligned}
\left(\int_{U_{j+1}} u^{p-1+\lambda_{j+1}} \mathrm{~d} z\right)^{\frac{1}{\gamma^{j+1}}} & \leq Y^{1+\frac{1}{\gamma}+\ldots+\frac{1}{\gamma^{j}}}(2 \gamma)^{p\left(\frac{1}{\gamma}+\frac{2}{\gamma^{2}}+\ldots+\frac{j}{\gamma^{j}}\right)} \int_{U_{0}} u^{p} \mathrm{~d} z \\
& \leq C Y^{\frac{N}{p}+1} \int_{U_{0}} u^{p} \mathrm{~d} z,
\end{aligned}
$$

so that

$$
\left(\int_{U_{j+1}} u^{p-1+\lambda_{j+1}} \mathrm{~d} z\right)^{\frac{1}{p-1+\lambda} j+1} \leq\left(C Y^{\frac{N}{p}+1} \int_{U_{0}} u^{p} \mathrm{~d} z\right)^{\frac{\gamma^{j+1}}{p-1+\lambda j+1}}
$$

Since $\gamma^{j+1} /\left(p-1+\lambda_{j+1}\right) \rightarrow 1 / 2$ and $p-1+\lambda_{j+1} \rightarrow \infty$ as $j \rightarrow \infty$, we obtain that

$$
\underset{Q(s)}{\operatorname{ess} \sup } u \leq C\left((S-s)^{-N-p} \int_{Q(S)} u^{p} \mathrm{~d} z\right)^{1 / 2}
$$


where $Q(s)=B\left(x_{0}, s\right) \times\left(t_{0}-(s / S)^{p} T, t_{0}\right)$. By Young's inequality, we have for every $1<\delta<2$ that

$$
\begin{aligned}
\underset{Q(s)}{\operatorname{ess} \sup u} & \leq\left(\underset{Q(S)}{\operatorname{ess} \sup ^{2-\delta}} u^{2}(S-s)^{-N-p} \int_{Q(S)} u^{p-2+\delta} \mathrm{d} z\right)^{1 / 2} \\
& \leq \frac{1}{2} \underset{Q(S)}{\operatorname{ess} \sup } u+\left((S-s)^{-N-p} \int_{B_{R}\left(x_{0}\right) \times\left(t_{0}-T, t_{0}\right)} u^{p-2+\delta} \mathrm{d} z\right)^{1 / \delta} .
\end{aligned}
$$

A standard iteration argument such as [9, Lemma 1.1] now finishes the proof. Indeed, if $f:\left[T_{0}, T_{1}\right] \rightarrow \mathbb{R}$ is a non-negative bounded function such that all $T_{0} \leq t \leq \tau \leq T_{1}$ satisfy

$$
f(t) \leq \theta f(\tau)+(\tau-t)^{-\eta} A,
$$

where $A, \theta, \eta \geq 0$ with $\theta<1$, then

$$
f\left(T_{0}\right) \leq C(\eta, \theta)\left(T_{1}-T_{0}\right)^{-\eta} A .
$$

Selecting $T_{0}:=\sigma R, T_{1}:=(\sigma R+R) / 2$ and the other variables so that (5.8) implies (5.9), we get the desired estimate.

Next, we consider the case where the non-negative subsolution is not necessarily bounded away from zero. Observe that the estimate differs from the usual estimate for the $p$-Laplacian because of the power $1 /(p-1)$ in the first term (cf. [7, Theorem 4.1] or [17, Theorem 3.4]). However, we have the additional assumption (5.10).

Theorem 5.3. Assume that $p \geq 2$ and that (G2) holds. Suppose that $u$ is a nonnegative weak subsolution to (1.1) in $\Xi$ and $B_{R}\left(x_{0}\right) \times\left(t_{0}-T, t_{0}\right) \Subset \Xi$ with $R, T<1$ such that

$$
\frac{R^{p}}{T} \leq 1
$$

Then, there exists a constant $C=C\left(N, p, C_{f}, \delta\right)$ such that we have the estimate

$$
\begin{aligned}
& \underset{B\left(x_{0}, R / 2\right) \times\left(t_{0}-T / 2^{p}, t_{0}\right)}{\operatorname{ess} \sup } u \\
& \leq C\left(\frac{R^{p}}{T}\right)^{\frac{1}{p-1} \cdot \frac{\delta-1}{\delta}}+C\left(\frac{T}{R^{p}} \int_{t_{0}-T}^{t_{0}} f_{B_{R}\left(x_{0}\right)} u^{p-2+\delta} \mathrm{d} x \mathrm{~d} t\right)^{\frac{1}{\delta}}
\end{aligned}
$$

for all $1<\delta<2$.

Proof. We denote

$$
\Lambda:=(1-\sigma)^{-N-p}, \theta:=\left(\frac{R^{p}}{T}\right)^{\frac{1}{p-1}}
$$


Using Lemma 5.2 on the subsolution $v:=\theta+u$, we get the estimate

$$
\begin{aligned}
& \operatorname{ess}_{B_{\sigma R\left(x_{0}\right) \times\left(t_{0}-\sigma^{p} T, t_{0}\right)} u \leq}\left(\Lambda \frac{T}{R^{p}} f_{B_{R}\left(x_{0}\right) \times\left(t_{0}-T, t_{0}\right)}(\theta+u)^{p-2+\delta} \mathrm{d} z\right)^{\frac{1}{\delta}} \\
& \leq C \Lambda^{\frac{1}{\delta}}\left(\frac{T}{R^{p}} \theta^{p-2+\delta}\right)^{\frac{1}{\delta}} \\
&+C \Lambda^{\frac{1}{\delta}}\left(\frac{T}{R^{p}} \int_{B_{R}\left(x_{0}\right) \times\left(t_{0}-T, t_{0}\right)} u^{p-2+\delta} \mathrm{d} z\right)^{\frac{1}{\delta}},
\end{aligned}
$$

where

$$
\frac{T}{R^{p}} \theta^{p-2+\delta}=T^{1-\frac{p-2+\delta}{p-1}} R^{-p+\frac{p(p-2+\delta)}{p-1}}=\left(T^{1-\delta} R^{p(\delta-1)}\right)^{\frac{1}{p-1}}=\left(\frac{R^{p}}{T}\right)^{\frac{\delta-1}{p-1}} .
$$

Taking $\sigma=1 / 2$ now yields the desired inequality.

Lemma 5.4. Assume that $p \geq 2$ and that $f(0)=0$. Let $u$ be a weak subsolution to (1.1) in $\Omega_{t_{1}, t_{2}}$. Then, $u_{+}=\max (u, 0)$ is also a weak subsolution.

Proof. Fix a non-negative test function $\zeta \in C_{0}^{\infty}\left(\Omega_{t_{1}, t_{2}}\right)$. We test the regularized equation in Lemma 3.1 with $\min \left\{k\left(u^{\epsilon}\right)_{+}, 1\right\} \zeta$. Then, by similar arguments as in the proof of Lemma 5.1 we get the estimate

$$
\begin{aligned}
& \int_{\Omega_{t_{1}, t_{2}}} \min \left\{k u_{+}, 1\right\}\left(-u \partial_{t} \zeta+|D u|^{p-2} D u \cdot D \zeta-\zeta f(D u)\right) \mathrm{d} z \\
& \quad \leq-\frac{1}{2 k} \int_{\Omega_{t_{1}, t_{2}}}\left(\min \left\{k u_{+}, 1\right\}\right)^{2} \partial_{t} \zeta \mathrm{d} z-k \int_{\{0<k u<1\}} \zeta|D u|^{p} \mathrm{~d} z .
\end{aligned}
$$

Letting $k \rightarrow \infty$ this implies

$$
\int_{\{u>0\}}-u \partial_{t} \zeta+|D u|^{p-2} D u \cdot D \zeta-\zeta f(D u) \mathrm{d} z \leq 0 .
$$

Since $f(0)=0$ and $u_{+} \partial_{t} \zeta=0=D u_{+}$a.e. in $\{u \leq 0\}$, we get that

$$
\int_{\Omega_{t_{1}, t_{2}}}-u_{+} \partial_{t} \zeta+\left|D u_{+}\right|^{p-2} D u_{+} \cdot D \zeta-\zeta f\left(D u_{+}\right) \mathrm{d} z \leq 0 .
$$

Theorem 5.5. Assume that $p \geq 2$, (G2) holds and that $f(0)=0$. Suppose that $u$ is a weak supersolution to (1.1) in $\Xi$. Let $u_{*}$ denote the lower semicontinuous regularization of $u$, that is,

$$
u_{*}(x, t):=\underset{(y, s) \rightarrow(x, t)}{\operatorname{ess} \liminf } u(y, s):=\lim _{R \rightarrow 0} \underset{B_{R}(x) \times\left(t-R^{p}, t+R^{p}\right)}{\operatorname{essinf}} u .
$$

Then, $u=u_{*}$ almost everywhere. 
Proof. For all $M \in \mathbb{N}$, we define the cylinders

$$
Q_{R}^{M}(x, t):=B_{R}(x) \times\left(t-M R^{p}, t+M R^{p}\right) .
$$

We denote by $E_{M}$ the set of Lebesgue points with respect to the basis $\left\{Q_{R}^{M}\right\}$, that is,

$$
E_{M}:=\left\{(x, t) \in \Xi: \lim _{R \rightarrow 0} f_{Q_{R}^{M}(x, t)}|u(x, t)-u(y, s)|^{p-\frac{1}{2}} \mathrm{~d} y \mathrm{~d} s=0\right\} .
$$

Then, $E_{M} \subset E_{M+1}$ so that

$$
E:=\bigcap_{M \in \mathbb{N}} E_{M}=E_{1} .
$$

Moreover, we have $|E|=|\Xi|$, which follows from [26, p. 13] by a simple argument, see for example [8, p. 54].

We now claim that if $\left(x_{0}, t_{0}\right) \in E$, then

$$
u\left(x_{0}, t_{0}\right) \leq \underset{(x, t) \rightarrow\left(x_{0}, t_{0}\right)}{\operatorname{ess} \liminf _{n}} u(x, t) .
$$

We make the counter assumption

$$
u\left(x_{0}, t_{0}\right)-\underset{(x, t) \rightarrow\left(x_{0}, t_{0}\right)}{\operatorname{ess} \liminf _{3}} u(x, t)=\varepsilon>0 .
$$

Let $R_{0}$ be a radius such that

$$
\left|\underset{(x, t) \rightarrow\left(x_{0}, t_{0}\right)}{\operatorname{ess} \liminf _{R}} u(x, t)-\underset{Q_{R}^{1}\left(x_{0}, t_{0}\right)}{\operatorname{essinf}} u\right| \leq \varepsilon / 2
$$

for all $0<R \leq R_{0}$. For such $R$, we have

$$
u\left(x_{0}, t_{0}\right)-\underset{Q_{R}^{1}\left(x_{0}, t_{0}\right)}{\operatorname{ess} \inf } u \geq \varepsilon / 2 .
$$

We set $v:=\left(u\left(x_{0}, t_{0}\right)-u\right)_{+}$. Since $\left(x_{0}, t_{0}\right) \in E$, we find for any $M \in \mathbb{N}$ a radius $R_{1}=R_{1}(M)$ such that

$$
\int_{Q_{R_{1}}^{M}\left(x_{0}, t_{0}\right)} v^{p-\frac{1}{2}} \mathrm{~d} x \mathrm{~d} t \leq f_{Q_{R_{1}}^{M}\left(x_{0}, t_{0}\right)}\left|u\left(x_{0}, t_{0}\right)-u\right|^{p-\frac{1}{2}} \mathrm{~d} x \mathrm{~d} t \leq\left(\frac{1}{M}\right)^{2} .
$$

On the other hand, by Lemma 5.4 the function $v$ is a weak subsolution to

$$
\partial_{t} v+\Delta_{p} v-g(D v) \leq 0
$$

where $g(\xi)=-f(-\xi)$. Observe also that the cylinder $Q_{R_{1}}^{M}\left(x_{0}, t_{0}\right)$ satisfies the condition (5.10) since $R_{1}^{p} /\left(M R_{1}^{p}\right) \leq 1$. Hence, we may apply Theorem 5.3 with $\delta=3 / 2$ and then use (5.13) to get

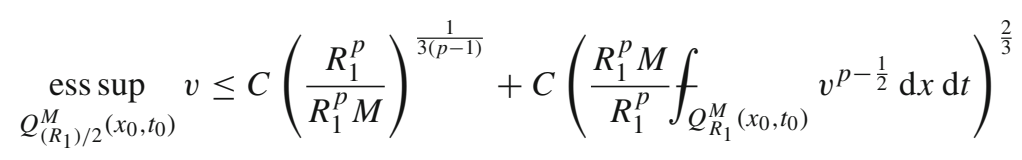




$$
\begin{aligned}
& \leq \frac{C}{M^{3(p-1)}}+C\left(M \cdot \frac{1}{M^{2}}\right)^{\frac{2}{3}} \\
& \leq C\left(\frac{1}{M}\right)^{\frac{1}{3}}
\end{aligned}
$$

Now we first fix $M$ so large that $C / M^{\frac{1}{3}} \leq \varepsilon / 4$ and this will also fix $R_{1}$. Then, we take $R \in\left(0, R_{0}\right]$ so small that $Q_{R}^{1}\left(x_{0}, t_{0}\right) \subset Q_{\left(R_{1}\right) / 2}^{M}\left(x_{0}, t_{0}\right)$. Then, (5.12) leads to a contradiction since

$$
\varepsilon / 4 \geq \underset{Q_{\left(R_{1}\right) / 2}^{M}\left(x_{0}, t_{0}\right)}{\operatorname{ess} \sup } v \geq \underset{Q_{R}^{1}\left(x_{0}, t_{0}\right)}{\operatorname{ess} \sup } v \geq u\left(x_{0}, t_{0}\right)-\underset{Q_{R}^{1}\left(x_{0}, t_{0}\right)}{\operatorname{essinf}} u \geq \varepsilon / 2 .
$$

Hence, (5.11) holds and we have

$$
u\left(x_{0}, t_{0}\right) \leq \underset{(x, t) \rightarrow\left(x_{0}, t_{0}\right)}{\operatorname{ess} \liminf _{n}} u(x, t) \leq \lim _{R \rightarrow 0} f_{Q_{R}^{1}} u(x, t) \mathrm{d} x \mathrm{~d} t=u\left(x_{0}, t_{0}\right) .
$$

Thus, $u_{*}=u$ almost everywhere and it is easy to show that $u_{*}$ is lower semicontinuous.

Funding Open Access funding provided by University of Jyväskylä (JYU).

Open Access. This article is licensed under a Creative Commons Attribution 4.0 International License, which permits use, sharing, adaptation, distribution and reproduction in any medium or format, as long as you give appropriate credit to the original author(s) and the source, provide a link to the Creative Commons licence, and indicate if changes were made. The images or other third party material in this article are included in the article's Creative Commons licence, unless indicated otherwise in a credit line to the material. If material is not included in the article's Creative Commons licence and your intended use is not permitted by statutory regulation or exceeds the permitted use, you will need to obtain permission directly from the copyright holder. To view a copy of this licence, visit http://creativecommons.org/licenses/ by/4.0/.

Publisher's Note Springer Nature remains neutral with regard to jurisdictional claims in published maps and institutional affiliations.

\section{REFERENCES}

[1] A. Attouchi, M. Parviainen, and E. Ruosteenoja. $C^{1, \alpha}$ regularity for the normalized $p$-Poisson problem. J. Math. Pures Appl., 108(4):553-591, 2017.

[2] A. Attouchi. Well-posedness and gradient blow-up estimate near the boundary for a Hamilton-Jacobi equation with degenerate diffusion. J. Diff. Eq., 253(8):2474-2492, 2012.

[3] J. Benedikt, P. Girg, L. Kotrla, and P. Takáč. Nonuniqueness and multi-bump solutions in parabolic problems with the $p$-Laplacian. J. Diff. Eq., 260(2):991-1009, 2016.

[4] V. Bobkov and P. Takáč. On maximum and comparison principles for parabolic problems with the $p$-Laplacian. To appear in RACSAM.

[5] V. Bobkov and P. Takáč. A strong maximum principle for parabolic equations with the $p$-Laplacian. J. Math. Anal. Appl., 419(1):218-230, 2014.

[6] M. G. Crandall, H. Ishii, and P.-L. Lions. User's guide to viscosity solutions of second order partial differential equations. Bull. Amer. Math. Soc., 27(1):1-67, 1992. 
[7] E. DiBenedetto. Degenerate parabolic equations. Springer-Verlag, 1993.

[8] L. C. Evans and R. F. Gariepy. Measure theory and fine properties of functions. CRC Press, revised edition, 2015.

[9] M. Giaquinta and E. Giusti. On the regularity of the minima of variational integrals. Acta Math., 148(1):31-46, 1982.

[10] H. Ishii. On the equivalence of two notions of weak solutions, viscosity solutions and distribution solutions. Funkcialaj Ekvacioj, 38:101-120, 1995.

[11] V. Julin and P. Juutinen. A new proof for the equivalence of weak and viscosity solutions for the p-Laplace equation. Comm. Partial Differential Equations, 37(5):934-946, 2012.

[12] P. Juutinen, P. Lindqvist, and J.J. Manfredi. On the equivalence of viscosity solutions and weak solutions for a quasi-linear equation. SIAM J. Math. Anal., 33(3):699-717, 2001.

[13] Z. Junning. Existence and nonexistence of solutions for $u_{t}=\operatorname{div}\left(|\nabla u|^{p-2} \nabla u\right)+f(\nabla u, u, x, t)$. J. Math. Anal. Appl., 172(1):130-146, 1993.

[14] N. Katzourakis. An Introduction To Viscosity Solutions for Fully Nonlinear PDE with Applications to Calculus of Variations in $L^{\infty}$. Springer, 2015.

[15] R. Korte, T. Kuusi, and M. Parviainen. A connection between a general class of superparabolic functions and supersolutions. J. Evol. Eq., 10(1):1-20, 2010.

[16] T. Kilpeläinen and P. Lindqvist. On the Dirichlet boundary value problem for a degenerate parabolic equation. SIAM J. Math. Anal., 27(3):661-683, 1996.

[17] T. Kuusi. Lower semicontinuity of weak supersolutions to nonlinear parabolic equations. Differential Integral Equations, 22(11-12):1211-1222, 2009.

[18] P. Lindqvist. Regularity of supersolutions. In Regularity estimates for nonlinear elliptic and parabolic problems, volume 2045 of Lecture Notes in Math, pages 73-131. 2012.

[19] P. Lindqvist. Notes on the p-Laplace equation (second edition). Univ. Jyväskylä, Report 161, 2017.

[20] P. Lindqvist and J. J. Manfredi. Viscosity supersolutions of the evolutionary $p$-Laplace equation. Differential Integral Equations, 20(11):1303-1319, 2007.

[21] R. Miculescu. Approximation of continuous functions by Lipschitz functions. Real Anal. Exchange, 26(1):449-452, 2000.

[22] M. Medina and P. Ochoa. On viscosity and weak solutions for non-homogeneous $p$-Laplace equations. Adv. Nonlinear Anal., 8(1):468-481, 2019.

[23] P. Pucci and J. Serrin. The maximum principle, volume 73 of Progress in non-linear differential equations and their applications. Birkhäuser, Boston, 2007.

[24] M. Parviainen and J. L. Vázquez. Equivalence between radial solutions of different parabolic gradient-diffusion equations and applications. To appear in Ann. Scuola Norm. Sup. Pisa Cl. Sci.

[25] J. Siltakoski. Equivalence of viscosity and weak solutions for the normalized $p(x)$-Laplacian. Calc. Var. Partial Differential Equations, 57(95), 2018.

[26] E. M. Stein. Harmonic analysis: Real-variable methods, orthogonality and oscillatory integrals. Princeton University Press, 1993.

\author{
Jarkko Siltakoski \\ Department of Mathematics and Statistics \\ University of Jyväskylä \\ P.O. Box 35 \\ 40014 Jyvaskyla \\ Finland \\ E-mail: jarkko.j.m.siltakoski@jyu.fi
}

Accepted: 19 December 2020 